\title{
Effective use of nanocarriers as drug delivery systems for the treatment of selected tumors
}

REVIEW

\author{
This article was published in the following Dove Press journal: \\ International Journal of Nanomedicine \\ 5 October 2017 \\ Number of times this article has been viewed
}

\author{
Fakhar ud Din ${ }^{1, *}$ \\ Waqar Aman ${ }^{2}, *$ \\ Izhar Ullah ${ }^{3}$ \\ Omer Salman Qureshi ${ }^{4}$ \\ Omer Mustapha ${ }^{5}$ \\ Shumaila Shafique ${ }^{6}$ \\ Alam Zeb ${ }^{7}$ \\ 'Department of Pharmacy, Quaid- \\ i-Azam University, Islamabad, \\ 2Department of Pharmacy, Kohat \\ University of Science and Technology, \\ Kohat, ${ }^{3}$ Department of Health and \\ Medical Sciences, University of \\ Poonch, Rawalakot, Azad Kashmir, \\ ${ }^{4}$ Faculty of Pharmacy, University of \\ Lahore, Lahore, ${ }^{5}$ Faculty of Pharmacy, \\ Ziauddin University, Karachi, \\ ${ }^{6}$ Department of Pharmaceutics, \\ Faculty of Pharmaceutical Sciences, \\ Dow University of Health Sciences, \\ Karachi, ${ }^{7}$ Riphah Institute of \\ Pharmaceutical Sciences, Riphah \\ International University, Islamabad, \\ Pakistan \\ *These authors contributed equally \\ to this work
}

\begin{abstract}
Nanotechnology has recently gained increased attention for its capability to effectively diagnose and treat various tumors. Nanocarriers have been used to circumvent the problems associated with conventional antitumor drug delivery systems, including their nonspecificity, severe side effects, burst release and damaging the normal cells. Nanocarriers improve the bioavailability and therapeutic efficiency of antitumor drugs, while providing preferential accumulation at the target site. A number of nanocarriers have been developed; however, only a few of them are clinically approved for the delivery of antitumor drugs for their intended actions at the targeted sites. The present review is divided into three main parts: first part presents introduction of various nanocarriers and their relevance in the delivery of anticancer drugs, second part encompasses targeting mechanisms and surface functionalization on nanocarriers and third part covers the description of selected tumors, including breast, lungs, colorectal and pancreatic tumors, and applications of relative nanocarriers in these tumors. This review increases the understanding of tumor treatment with the promising use of nanotechnology.

Keywords: nanocarriers, drug delivery, solid lipid nanoparticles, liposomes, polymeric nanoparticles, dendrimers, breast tumor, lungs tumor, colorectal tumor, prostate tumor
\end{abstract}

\section{Introduction to nanocarriers}

Nanocarriers are colloidal drug carrier systems having submicron particle size typically $<500 \mathrm{~nm} .{ }^{1}$ Nanocarriers have been extensively investigated in the past few decades as they showed great promise in the area of drug delivery. Nanocarriers, owing to their high surface area to volume ratio, have the ability to alter basic properties and bioactivity of drugs. Improved pharmacokinetics and biodistribution, decreased toxicities, improved solubility and stability, controlled release and site-specific delivery of therapeutic agents are some of the features that nanocarriers can incorporate in drug delivery systems. ${ }^{2,3}$ Moreover, the physiochemical properties of nanocarriers can be tuned by altering their compositions (organic, inorganic or hybrid), sizes (small or large), shapes (sphere, rod or cube) and surface properties (surface charge, functional groups, PEGylation or other coating, attachment of targeting moieties). ${ }^{4}$ The overall goal of utilizing nanocarriers in drug delivery is to treat a disease effectively with minimum side effects. ${ }^{2}$

Anticancer chemotherapeutics when delivered via conventional drug delivery systems present a number of unique problems, including poor specificity, high toxicity and induction of drug resistance. ${ }^{5}$ These obstacles decrease the therapeutic value of many anticancer drugs. Nanocarrier-based platforms have enabled effective delivery of anticancer drugs into the tumors by exploiting the pathophysiology of tumor microenvironment, thereby significantly improving the therapeutic outcomes 
for oncological conditions. ${ }^{6}$ Furthermore, the overexpressed receptors on tumor cells surface have also been targeted with nanocarriers platforms decorated with targeting ligands. A number of nanocarrier-based products have been approved for the treatment of various tumors, and many others are in different phases of clinical trials. ${ }^{4}$ In the current review, we first discussed features of different types of nanocarriers (organic, inorganic and hybrid) along with their relevance in cancer therapy. We then elaborated the targeting mechanisms and surface functionalization on nanocarriers to improve their targetability. Finally, we discussed selected tumors and application of nanocarriers in the respective tumor.

\section{Organic nanocarriers}

\section{Solid lipid nanoparticles (SLNs)}

SLNs, developed in early 1990s, are nanosized colloidal drug carriers in the size range of 50-1,000 nm. ${ }^{7}$ SLNs are prepared by dispersing melted solid lipid(s) in water, whereas emulsifier(s) are used to stabilize the dispersion. The two most commonly used methods for preparing SLNs are high pressure homogenization and microemulsification. SLNs provide a highly lipophilic lipid matrix for drugs to be dispersed or dissolved. ${ }^{8}$ A wide variety of solid lipids including mono-, di- and triglycerides; free fatty acids; free fatty alcohols; waxes and steroids have been utilized for the preparation of SLNs. SLNs are quite similar to nanoemulsions except that different kinds of lipids are used in both the formulations. Lipids that are solid at room temperature are used in SLNs instead of liquid lipids (oils) used in nanoemulsions. ${ }^{9}$

SLNs as nanocarriers offer a number of advantages over their colloidal counterparts, including nanoemulsions, liposomes and polymeric nanoparticles (PNPs). Some of the areas where SLNs score better than their counterparts include controlled drug delivery, lack of biotoxicity, high drug payload, improved bioavailability of poorly water-soluble drugs, better stability and easy as well as economical large-scale production. ${ }^{10,11}$ Various models have been proposed to incorporate drugs into SLNs. Depending upon the composition of SLNs (lipid, drug and surfactant) and production conditions (hot or cold homogenization), drug can either be dispersed homogeneously in lipid matrix (solid solution/homogeneous matrix model) of SLNs (Figure 1), incorporated into the shell surrounding the lipid core (drug-enriched shell model) or incorporated into the core surrounded by a lipid shell (drugenriched core model). ${ }^{12}$

Since their development, SLNs have been extensively used as a carrier for numerous antitumor chemotherapeutic moieties. The versatile and favorable properties of SLNs

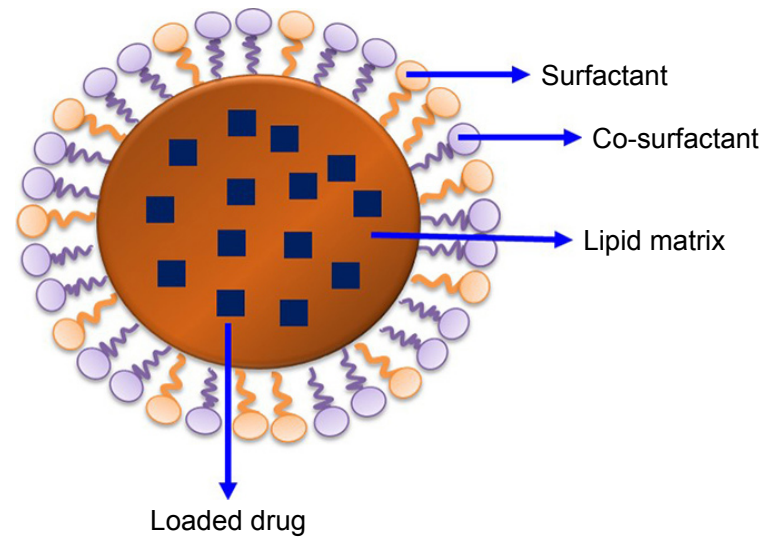

Figure I Schematics of SLN.

Abbreviation: SLN, solid lipid nanoparticle.

make them promising nanocarriers to overcome or at least minimize the drawbacks of conventional chemotherapy. SLNs provide a natural platform to incorporate lipophilic anticancer drugs. Moreover, recent advances in the field of SLNs (eg, polymer-lipid hybrid type of SLNs, lipid-drug conjugate nanoparticles [NPs]) made it possible to incorporate hydrophilic and ionic anticancer drugs as well. However, quick elimination of SLNs from the blood flow by reticule endothelial system (RES), encapsulation of hydrophilic and ionic anticancer drugs and controlling the rate and extent of drug release from SLNs are the major obstacles faced by SLNs that restrain them from becoming effective nanocarriers in anticancer drug delivery. All of these aspects of SLNs as anticancer drug carriers have been excellently reviewed by Wong et al. ${ }^{5}$ Few examples where SLN systems have been utilized for the delivery of anticancer drugs are docetaxel, ${ }^{13}$ doxorubicin, ${ }^{14}$ paclitaxel,,${ }^{15}$ methotrexate ${ }^{16}$ and 5-fluorouracil (5-FU). ${ }^{17}$

\section{Liposomes}

Liposomes attained a great attention during the last few decades in biomedicine, especially as a drug delivery system for antitumor drugs. ${ }^{18}$ They showed a number of advantages over conventional systems which are, but not limited to, enhanced delivery of drug, protection of active drug from environmental factors, improved performance features of the product, preventing early degradation of the encapsulated drug, cost-effective formulations of expensive drugs and efficient treatment with reduced systemic toxicity. ${ }^{19}$ Moreover, drugs associated with liposomes have markedly altered pharmacokinetic properties compared to free drugs in solution. ${ }^{8}$ They can be covered with polymers such as polyethylene glycol (PEG; PEGylated or stealth liposomes) to exhibit prolonged half-life in blood circulation. Liposomes are spherical 
vesicles having an aqueous core enclosed by lipid bilayers. They have single or multiple bilayered membrane assembly formed of natural or synthetic lipids (Figure 2). Those containing one bilayer membrane are termed as small unilamellar vesicles or large unilamellar vesicles based on their sizes. If more than one bilayer is present, then they are called as multilamellar vesicles. Liposomes vary with respect to composition, size, surface charge and method of preparation..$^{20}$ Liposomes are commonly used as model cells or carriers for various bioactive agents including drugs, vaccines, cosmetics and nutraceuticals. ${ }^{19}$

The biodegradable and biocompatible composition of liposomes made them excellent therapeutic carriers. Moreover, their distinctive capacity to accommodate both watersoluble and lipid-soluble agents, correspondingly in their aqueous central part and lamellae, has increased their use in biomedicine formulations. ${ }^{20}$ Different polymers are used to enhance their stability and half-life, which include PEG and poly(lactic-co-glycolic acid) (PLGA). ${ }^{19}$ Drug-loaded liposome preparations control the drug distribution within plasma, thus leading to improved biodistribution and pharmacokinetics of drugs. For instance, doxorubicin-loaded PEGylated liposome decreases the volume of doxorubicin distribution from 1,000 to $2.8 \mathrm{~L} / \mathrm{m}^{2}$ in plasma as compared to free drugs in solution. ${ }^{6}$ Moreover, it leads to a higher drug concentration inside tumor but reduces drug concentration in normal tissues. Liposomes can also be conjugated to antibodies or ligands in order to enhance target specificity. ${ }^{20}$

\section{Dendrimers}

Dendrimers are frequently branched macromolecules with various arms originating from the central core. ${ }^{21}$ Usually, they are produced by using natural or synthetic components, which include sugars, nucleotides and amino acids. Their stepwise synthesis enables them to adjust molecules with exceedingly regular branching pattern, a distinctive molecular weight and

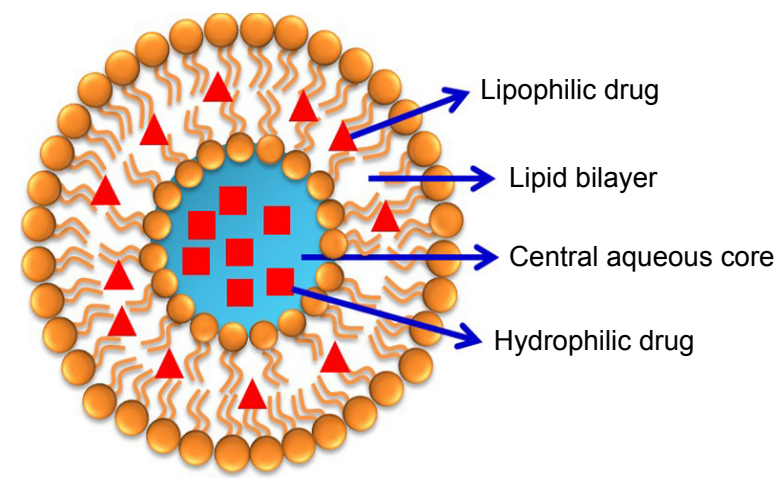

Figure 2 Diagrammatic representation of liposome structure. a distinct number of peripheral groups. Dendrimers obtained by stepwise synthetic techniques are distinctive as compared to those produced by polymerization processes because of the well-arranged and irregular branching patterns, respectively. ${ }^{21}$

Dendrimers are the most likely drug delivery systems owing to their inimitable characteristics, including distinctive molecular weight, increased number of branching, multivalency, spherical shapes and monodispersed macromolecules with an average diameter of $1.5-14.5 \mathrm{~nm} .{ }^{22}$ A typical dendrimer molecule comprises exceedingly branched layers consisting of reiterating units, numerous active terminal groups and an initiator core. Their architectural design offers an extraordinary control over the dendrimer shape, size, branching length and surface functionality. The geographically remote core located inside the dendrimer is a fascinating characteristic of dendrimers, which indicates the site isolation in biomolecules. Drugs can be loaded to the cavities in the dendrimers cores through hydrogen bonds, chemical linkages or hydrophobic interactions. Each level of added branches to the core throughout the synthesis process is called as a generation. The exceedingly branched structure of dendrimers results in bulky exterior groups. The drugs and targeting ligands can be attached to transform the surface functionalities in order to obtain specific objectives, which usually implicate precise contact at cell walls and biologically active sites. The preclinical development of dendrimers has been focused largely on forming dendrimer-drug conjugates. Recently, dendrimers have been extensively used in fields of biomedicine, including gene delivery, immunology, magnetic resonance imaging, vaccines and antiviral, antibacterial and anticancer drug delivery. ${ }^{23} \mathrm{~A}$ descriptive structure of dendrimers composed of core, halfway layers and exterior groups is shown in Figure 3.

Dendrimer-drug conjugate is formed when the drug is covalently bonded to a dendrimer at the core or on the terminal

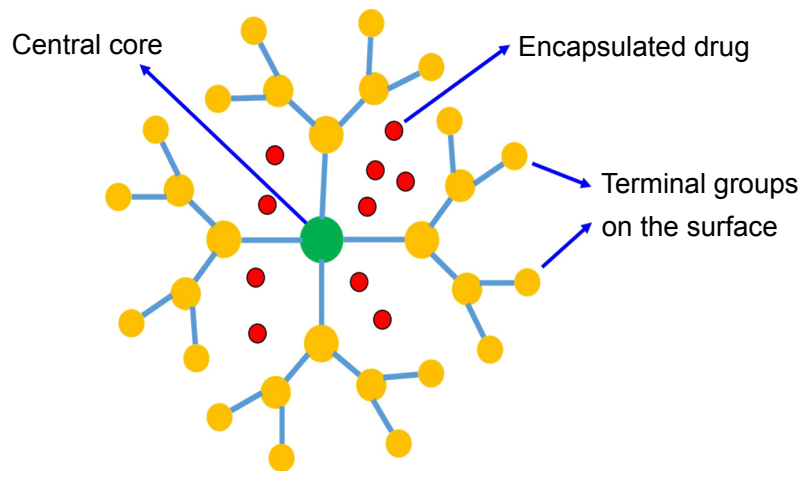

Figure 3 Structure of dendrimers. 
groups and very infrequently in the inner layers, ie, at the branching points. The effective concentration of a drug is prominently amplified at the targeted site, if the drug is linked to numerous peripheral groups in a dendrimer. Primarily, this is beneficial for the use of prodrugs. Being monodispersed, structurally controlled macromolecules with a definite size and molecular weight, dendrimers-drug conjugate is a carrier of choice over conventional polymeric drug delivery carriers. The drug and dendrimer linker is of prime significance if the drug is attached to exterior groups of a dendrimer. This is because the drug is required to be released in an active form at the site of action. The drug is attached to the dendritic boundary with the help of acid labile or disulphide (susceptible to reduction) linkers. Usually, benefits are taken from acidic or reducing atmosphere adjacent to the cancerous cell. Thus, every drug molecule is distinctly cleaved over an extended period of time. In this respect, a sophisticated architecture/ design was described in which a single generating event at the core resulted in disassociation of the dendrimer leading to the release of all the attached paclitaxel molecules to the periphery. ${ }^{24}$ The drug release in these dendrimers is triggered by introducing a masked 4-aminocinnamyl alcohol linker that resulted in the reduction of the nitro group to amino group in a slightly acidic tumor environment.

Dendrimers were successfully used to enhance the efficiency of doxorubicin as described by Lai et al. They used photochemical internalization (PCI) technology, which is famous for abolishing the cytoplasmic membrane and enabling the proclamation of macromolecules trapped in cytoplasmic vesicles, leading to the increased cytotoxicity to cancerous tissue. ${ }^{25}$ Cisplatin-dendrimer conjugation was reported to have enhanced activity in vitro and in vivo as compared to free cisplatin after intraperitoneal administration into mice having B16F10 tumor cells. Similarly, after intravenous administration, dendrimer-platinate conjugation resulted in increased antitumor activity as compared to cisplatin alone. ${ }^{26}$ In an another attempt, Zhuo et $\mathrm{al}^{27}$ prepared 5-FU-dendrimer conjugates of different generations (0.5-5.5) using timesequenced propagation technique. These conjugates were found to have favorable controlled release characteristic for anticancer drugs. PEGylated-based dendrimers in conjugation with doxorubicin showed an enhancement in the blood circulation time, reduced toxicity and less accumulation of drug. Moreover, they prevent the development of Doxunresponsive C-26 tumor when administered subcutaneously into mice. ${ }^{28}$ Similarly, in another study, it was observed that PEGylated polyamidoamine (PAMAM) dendrimers in conjugation with 5-FU resulted in sustained release of antitumor drugs both in vitro and in vivo in albino rats. Additionally, this study resulted in abridged leakage and hemolytic toxicity. ${ }^{29}$

\section{PNPs}

In the last few decades, polymers have gained a great deal of attention in the area of drug delivery since they offer a number of attractive features in drug delivery. PNPs are solid, nanosized (10-1,000 nm) colloidal particles made up of biodegradable polymers. ${ }^{30,31}$ Based on their structural organization, PNPs can either be categorized as nanospheres (matrix type) or nanocapsules (reservoir type; Figure 4). Nanospheres type of PNPs disperse/entrap the drug in the polymer matrix, while in case of nanocapsules type of PNPs, the drug is dissolved/dispersed in liquid core of oil or water encapsulated by a solid polymeric membrane. In both types of PNPs, the adsorption or chemical conjugation of drug on to the surface (of the matrix or capsule) is also possible. ${ }^{32}$ A number of methods have been developed to prepare PNPs depending upon the composition and the desired properties of PNPs. These methods can be conveniently classified into two categories, namely, dispersion of preformed polymers and direct polymerization of monomers. The methods involving the dispersion of preformed polymers include solvent evaporation, salting out, nanoprecipitation, dialysis and supercritical fluid technology. The methods involving direct polymerization of monomers include emulsification polymerization, miniemulsion polymerization, microemulsion polymerization, interfacial polymerization and controlled/ living radical polymerization. All of these methods have been reviewed in detail by Rao and Geckeler. ${ }^{30}$

A number of biocompatible and biodegradable, both natural and synthetic, polymers have been utilized for the preparation of PNPs. Being biodegradable, these polymers are degraded into individual monomers inside the body and hence are removed from the body through normal metabolic pathways. ${ }^{2}$ Most commonly used synthetic polymers include

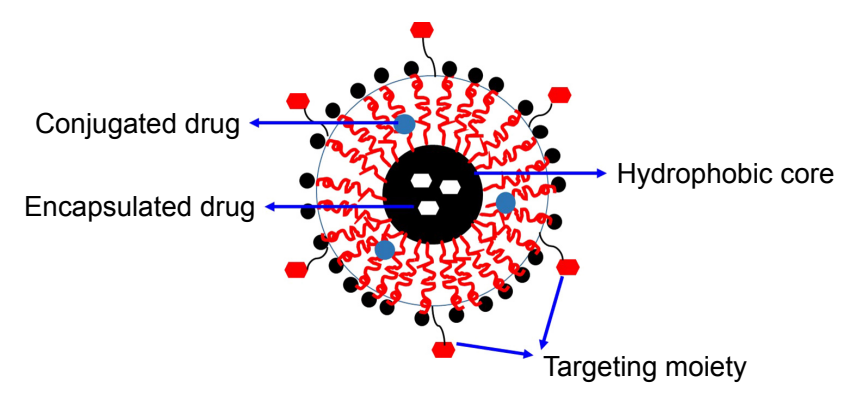

Figure 4 Schematics of PNPs.

Abbreviation: PNP, polymeric nanoparticle. 
polylactic acid (PLA), polyglycolic acid (PGA), PLGA, PEG, polycaprolactone (PCL), $N$-(2-hydroxypropyl)methacrylamide (HPMA) copolymer, polyaspartic acid (PAA) and polyglutamic acid. However, mostly used natural polymers include albumin, alginate, chitosan, collagen, dextran, gelatin and heparin..$^{33}$ Apart from the general salient features shared by all the nanocarriers in cancer therapy, PNPs offer better stability on storage and in vivo (in the blood), higher drug payload, more homogeneous particle size distribution, better and controllable physicochemical properties, higher drug circulation times and more controlled drug release compared with their colloidal counterparts such as polymeric micelles (PMs) and liposomes. ${ }^{34}$ All of these characteristics are highly desirable in the context of cancer treatment.

The earliest attempt to deliver an anticancer drug, doxorubicin, using PNPs was made in 1979. Since then a number of reports have been published for the delivery of anticancer chemotherapeutic agent via PNPs. PNPs are highly versatile with their design because they can be made with a wide range of polymers from natural and synthetic sources. Moreover, by controlling the physicochemical properties of polymers (eg, molecular weight, dispersity index, hydrophobicity and crystallinity), the degradation of PNPs and hence drug release can be accurately controlled. ${ }^{35}$ It is also possible to make multifunctional PNPs for cancer treatment having the desired sizes, shapes and surface modifications. ${ }^{36}$ Thanks to the advances in polymer sciences and engineering that made the development of smart polymer (stimuli-sensitive polymer) possible. These polymers are capable to alter their physicochemical characteristics in response to certain environmental signals, leading to more accurate and programmable drug delivery in cancer treatment. ${ }^{31}$ All these developments have given a new direction to the cancer treatment. Currently, a number of PNPs loaded with anticancer drugs are in the different phases of clinical trials. ${ }^{37}$ A plethora of articles are being published describing the positive aspects of PNPs encapsulating anticancer drugs. A few representative examples of studies using PNPs as a carrier for anticancer molecules include PLGA NPs, ${ }^{38,39}$ PLA NPs,${ }^{40}$ PCL NPs, ${ }^{39}$ chitosan $\mathrm{NPs}^{41}$ and alginate NPs. ${ }^{42}$

\section{PMs}

PMs are nanosized (10-100 nm) colloidal particles formed by the self-assembly of synthetic amphiphilic di- or tri-block copolymers in an aqueous milieu. ${ }^{36}$ Being amphiphilic in nature, di- or tri-block copolymers thus contain both hydrophobic and hydrophilic segments. These block copolymers when exposed to an aqueous environment, above certain concentration (called critical micelle concentration [CMC]), form micelles. The hydrophobic segment of block copolymer constitutes the core of micelle, while hydrophilic segment forms the shell of micelles. Therefore, PMs have a core/shell structure with a hydrophobic core and a hydrophilic shell (Figure 5). ${ }^{43}$ The hydrophobic core of PMs allows the entrapment of hydrophobic drugs and controls the drug release properties of PMs. However, the hydrophilic shell of PMs serves to stabilize the core, ensures the PMs' solubility in the aqueous milieu and controls in vivo pharmacokinetics. ${ }^{44}$ The drugs can either be incorporated into the PMs by physical entrapment or via chemical attachment. ${ }^{45}$ Some commonly used methods for the preparation of PMs are dialysis method, oil-in-water emulsion method, solvent evaporation method, co-solvent evaporation method and freeze-drying method. ${ }^{46}$

PMs offer promising nanocarriers for the anticancer drug delivery. As many of the anticancer drugs tend to be poorly water soluble (hydrophobic), PMs allow such hydrophobic anticancer drugs to be entrapped into their cores, thus significantly increasing their water solubility. Moreover, the hydrophilic shell of PMs causes them to have increased circulation times in blood by preventing the recognition and subsequent uptake of PMs by the RES. Thus, the smaller size $(10-100 \mathrm{~nm})$ and prolonged in vivo circulation times cause the PMs to preferentially accumulate in the tumor site by the enhanced permeability and retention (EPR) effect (passive targeting).$^{43}$ All of these effects improve the bioavailability and therapeutic effectiveness of hydrophobic anticancer drugs. Apart from passive targeting, the active targeting of cancers with PMs is also possible by designing the smarter PMs (environment/stimulus-responsive PMs, ie, responsive to changes in $\mathrm{pH}$, temperature, etc.) or by surface modification of PMs with tumor-targeting ligand. ${ }^{46}$ Advances in polymer sciences and versatility of PMs forming block copolymers make them an attractive carrier in the cancer therapy.

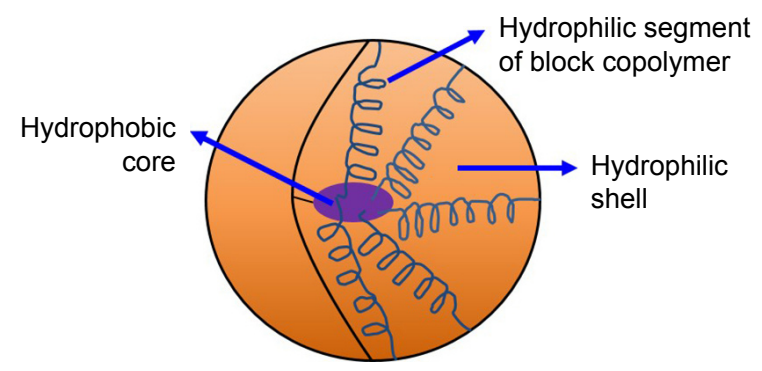

Figure 5 Structure of PMs.

Abbreviation: PM, polymeric micelle. 
A few commonly used block copolymers for PMs are poloxamers, PEGylated polylactic acid (PEG-PLA), PEGylated polyaspartic acid (PEG-PAA), PEGylated polyglutamic acid, PEGylated polycaprolactone (PEG-PCL) and PEGylated poly(lactic-co-glycolic acid) (PEG-PLGA). A number of PMs carrying anticancer drugs are currently in different phases of clinical trials. ${ }^{43}$ Many chemotherapeutic agents for cancer, including methotrexate, cisplatin, paclitaxel, docetaxel and doxorubicin, have successfully been formulated in PMs. A few representative studies describing PMs for drug delivery applications of cancer include poloxamer-based micelles, ${ }^{47}$ PEG-PLA micelles, ${ }^{48}$ PEGylated polyglutamic acid micelles ${ }^{49}$ and PEG-PAA micelles. ${ }^{45}$

\section{Virus-based nanoparticles (VNPs)}

VNPs or virus-like particles (VLPs) are nanosized (approximately $<100 \mathrm{~nm}$ ), self-assembled robust protein cages having uniform nanostructures and well-defined geometry (Figure 6). ${ }^{50,51}$ Recently, VNPs (viruses as nanocontainers) have been widely explored for nanotechnological purposes, including drug delivery, gene therapy, vaccination, imaging and targeting. ${ }^{52}$ VNPs or protein cages (ie, viruses) from different sources including plant viruses (cowpea chlorotic mottle virus [CCMV], cowpea mosaic virus [CPMV], red clover necrotic mosaic virus [RCNMV], tobacco mosaic virus [TMV]), insect viruses (flock house virus), bacterial viruses or bacteriophages (MS2, M13, Q $\beta$ ) and animal viruses (adenovirus, polyomavirus) have been investigated for nanotechnology and drug delivery applications..$^{50,51,53}$ As an arising nanocarrier platform, VNPs offer several attractive features including morphological uniformity, biocompatibility, easy surface functionalization and availability in a variety of sizes and shapes. ${ }^{53}$ Possibility of versatile chemical and genetic modifications on their surface enables VNPs to meet the

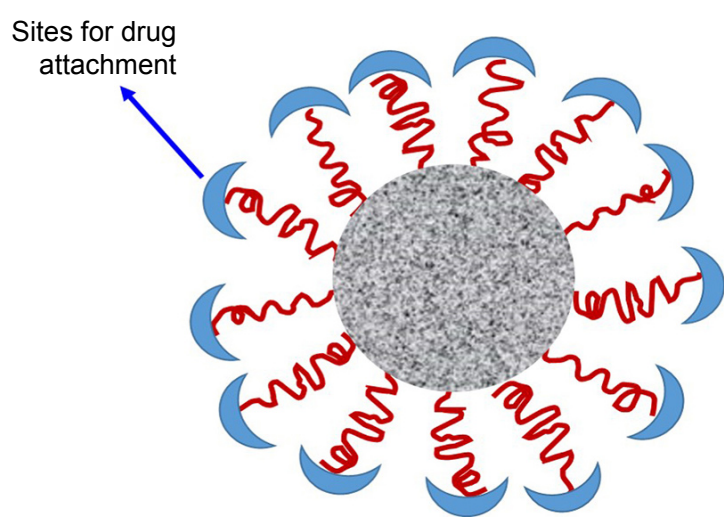

Figure 6 Structure of VNPs. Abbreviation: VNP, virus-based nanoparticle. requirements of drug nanocarriers including biocompatibility, hydrophilicity and enhanced drug entrapment proficiency. Additionally, PEGylating the surface of VNPs can improve their circulation time in the host. ${ }^{53,54}$

For drug delivery applications, drugs can either be physically entrapped in VNPs or chemically attached to the surface of VNPs. ${ }^{55}$ In the physical entrapment, a simple and natural process of supramolecular self-assembly/reassembly of viral protein capsid is utilized to load the drug cargo in VNPs. While in the chemical attachment, drug cargo is loaded onto VNPs via covalent attachment of drug molecules to certain (naturally existing or engineered) reactive sites on the capsid proteins. As a drug carrying nanocontainers, VNPs can be directed toward specific cancer targets either by exploiting the natural affinity of some viruses for receptors that are overexpressed in various tumors (eg, transferrin receptor [TfR]) or by modifying the exterior surface of virus nanocarriers through chemical or genetic procedures. ${ }^{56}$ VNPs loaded with chemotherapeutic agents have been investigated for tumor targeting in various studies. ${ }^{57,58}$

\section{Inorganic nanocarriers}

\section{Carbon nanotubes (CNTs)}

CNTs are nanosized, hollow, tube-like assemblies of carbon atoms discovered by Iijima ${ }^{59}$ in 1991 . CNTs belong to the family of fullerenes (a third allotropic form of carbon) and are formed by rolling up of graphene sheet(s) into a tube-like structure. ${ }^{60}$ CNTs can be classified as single-walled carbon nanotubes (SWCNTs) formed by rolling up a single graphene sheet or multi-walled carbon nanotubes (MWCNTs) formed by rolling up several concentric graphene sheets into a tubelike assembly (Figure 7A and B). CNTs have cross-sectional dimensions in nanometer range and lengths that can extend over a thousand times their diameters. Typically, the outer diameters of SWCNTs and MWCNTs are in the range of 0.4-2 $\mathrm{nm}$ and 2-100 nm, respectively. ${ }^{61}$ Some commonly
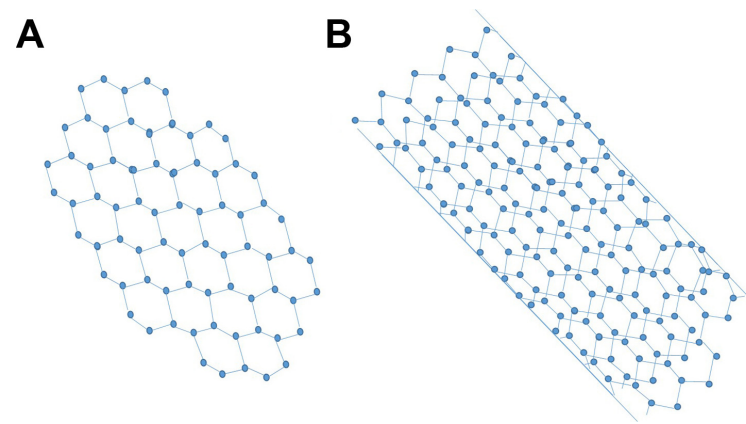

Figure 7 Graphical representation of SWCNTs (A) and double-walled CNTs (B). Abbreviations: CNT, carbon nanotube; SWCNT, single-walled carbon nanotube. 
used techniques for the production of CNTs include arc discharge, laser ablation and thermal or plasma-enhanced chemical vapor deposition. ${ }^{62}$

CNTs possess some distinctive physicochemical and biological characteristics that make them a promising carrier for drug delivery. Some of these characteristics include nanoneedle shape, hollow monolithic structure, high aspect ratio (length: diameter $>200: 1$ ), ultrahigh surface area, ultralight weight, high mechanical strength, high electrical and thermal conductivities and their ability for surface modifications. ${ }^{61,63}$ The needle-like shape of CNTs allows them to cross the cell membrane via endocytosis or "needle-like penetration" and subsequently enter into the cell. ${ }^{37}$ The major problems with CNTs as a drug carrier are their poor water solubility and toxicity. However, CNTs have the ability to be surface functionalized, which render them water soluble, biocompatible, non/less toxic and as a serum-stable carrier. ${ }^{64}$

Owing to their cell penetration abilities, unique physicochemical characteristics, high drug payload, intrinsic stability, structural flexibility and appropriate surface functionalization (for different purposes), CNTs are one of the ideal nanocarriers for cancer therapy. CNTs have been extensively studied in the past 2 decades as a carrier for anticancer drug delivery ${ }^{65}$ Anticancer drugs can either be encapsulated in the inner cavity of $\mathrm{CNTs}^{66}$ or can be attached, either covalently or noncovalently, to the surface of CNTs. ${ }^{67}$ Furthermore, the attachment of different targeting agents to the surface-functionalized CNTs allow targeted delivery of anticancer drug to the area of interest. ${ }^{68} \mathrm{~A}$ few examples of applications of CNTs in anticancer drug delivery include methotrexate, ${ }^{69}$ paclitaxel, ${ }^{70}$ doxorubicin,,${ }^{71,72}$ cisplatin, ${ }^{73,74}$ carboplatin $^{75}$ and mitomycin C..$^{76}$

\section{Mesoporous silica nanoparticles (MSNs)}

Silica $\left(\mathrm{SiO}_{2}\right)$ materials have got increased applications in the field of biomedicine owing to their simple synthesis procedures and availability for mass production. Among silica materials, mesoporous silicas are of particular importance in drug delivery as they are able to host large amounts of drugs by virtue of their honeycomb-like architecture with hundreds of pores (Figure 8). ${ }^{77}$ MSNs possess several attractive features such as good biocompatibility, large specific surface area and pore volume, high loading capacity, controllable pore diameters ranging from 2 to $50 \mathrm{~nm}$ with narrow pore size distribution, good thermal and chemical stability and versatility of loading drugs with hydrophilic and lipophilic characteristics, which make them promising nanoscale drug carriers. ${ }^{77,78}$

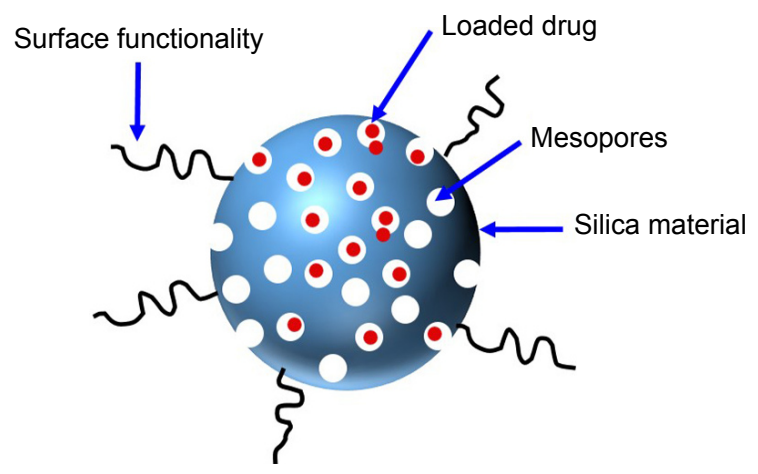

Figure 8 Schematics of MSNs.

Abbreviation: MSN, mesoporous silica nanoparticle.

In addition, ease of surface functionalization for controlled and targeted drug delivery enables MSNs to enhance therapeutic efficacy and reduce the toxicity of drugs. ${ }^{79}$

Unique architecture and attractive properties of MSNs place this class of nanocarriers at an ideal position for the delivery of anticancer drugs. Mesoporous structure of MSNs enables them to load large amount of anticancer drug, nanoscale particle size range aids them to accumulate in tumor tissues via passive targeting and convenient surface functionalization of MSNs with different site specific targeting agents enables them to target tumor tissues via active targeting mechanism. ${ }^{79}$ Many different anticancer drugs, including paclitaxel, ${ }^{80}$ camptothecin, ${ }^{81}$ doxorubicin ${ }^{82}$ and methotrexate, ${ }^{83}$ have been effectively delivered via MSNs.

\section{Organic/inorganic hybrid nanocarriers}

Organic/inorganic hybrid nanocarriers have been developed to combine the advantages of organic and inorganic materials. Specific functionalities of organic materials at the surface of inorganic NPs have been utilized to enhance the selectivity and efficiency of antitumor agents. For instance, surface coating with polyethyleneimine (PEI) not only enhances cellular uptake of MSNs but also generates cationic surface for efficient nucleic acid delivery. ${ }^{84}$ In another study, integration of hyperbranched PEI with MSNs resulted in a high payload and sustained intracellular delivery of short interfering RNA (siRNA). ${ }^{85}$ These MSNs/PEI hybrid nanocarriers successfully reached the tumor milieu followed by oozing from endosomes to the cytoplasm.

The meticulous construction of systems composed of lipid bilayers supported on solid material has attracted significant interest owing to their nanotechnological and biomaterial applications. MSNs/lipid bilayer hybrid nanocarriers are one of such systems where lipid bilayers are used to cap the channels of MSNs, in order to prevent premature 
release of loaded drug, circumvent multidrug resistance, prolong retention of hydrophilic drug cargo and achieve stimuli-responsive drug release. ${ }^{86,87}$ Desai et al ${ }^{86}$ reported the development of MSNs/lipid bilayer hybrid system with improved retention and intracellular delivery of zoledronic acid in breast cancer. In another study, Han et $\mathrm{al}^{87}$ developed doxorubicin-loaded, hybrid, lipid-capped MSNs with $\mathrm{pH}$ and redox-responsive release of drug cargo. These hybrid nanocarriers were able to release doxorubicin within the tumor cells and showed increased doxorubicin uptake efficiency, cytotoxicity and intracellular accumulation as compared to the free drug solution. ${ }^{87}$

\section{Targeting mechanisms and surface functionalization on nanocarriers \\ Passive mechanism}

Tumor-bearing blood vessels are leaky in nature, thereby permitting the nanocarriers to easily move into the interstitial space after crossing the endothelium barrier. Conditional to the tumor type, dimension of the tumor endothelial cell linings varies from 100 to $700 \mathrm{~nm}$, which is 50- to 70-fold more than the normal and distinctive endothelium (up to $10 \mathrm{~nm}$ ). ${ }^{88}$ Furthermore, solid tumors have poor lymphatic drainage system; thus, an inadequate circulatory repossession of the extravasated molecules occurs leading to the assemblage of nanocarriers at the tumor location. This is called as the EPR effect, ${ }^{89}$ which is believed as a great approach in effective tumor targeting. However, nanocarriers loaded with low molecular weight drugs reenter the blood circulation through the diffusion phenomenon and thus cannot stay at the tumor location for an extended period of time. The targeting effect of such drugs totally depends upon the pathophysiological and immunochemical characteristics of the tumor tissues, which are also known as "passive targeting" (Figure 9A). ${ }^{89}$ The use of nanocarriers not only enhances the systemic circulation of drugs but also improves the targeting of tumor cells utilizing the EPR effect. ${ }^{90}$ Various techniques are utilized to obtain the prolonged retention of drugs, including polymericbased and $\mathrm{pH}$-dependent nanocarrier systems.

Additionally, passive targeting is contributed by the distinctive and dissimilar microenvironment adjacent to the tumor cells as compared to normal cells. Quick spreading and hyperactive tumors have an extraordinarily increased metabolic ratio. Thus, the available oxygen and nutrients are not adequate, leading to attain extra energy through glycolysis by the tumor cells, causing an acidic environment. ${ }^{91}$ Furthermore, tumor cells discharge distinctive enzymes such as metalloproteases, which are associated with their movement and existence. ${ }^{92}$ For targeting these distinctive tumor microenvironment, various nanocarriers were used, including liposomes, polymers, micelles, NPs and antibodies. Moreover, a number of strategies have been adopted and explained for their possible mechanisms of passive and active drug targeting to tumor cells and endothelial cells and activated drug transport. A meaningful progress has been done in this filed as a good number of nanomedicines with passive
A

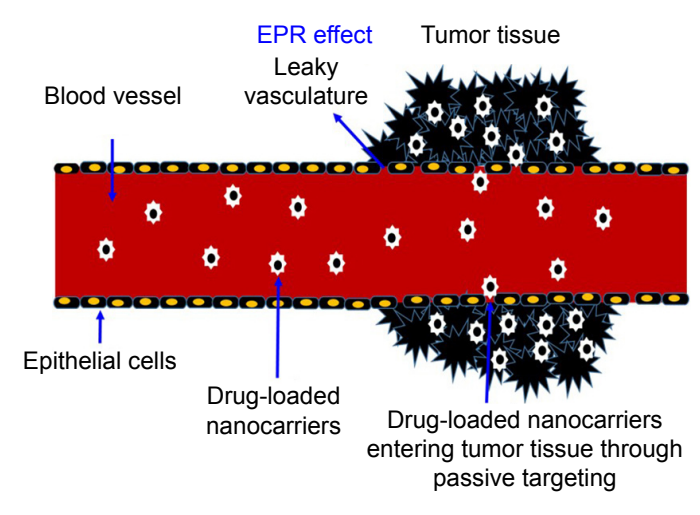

B
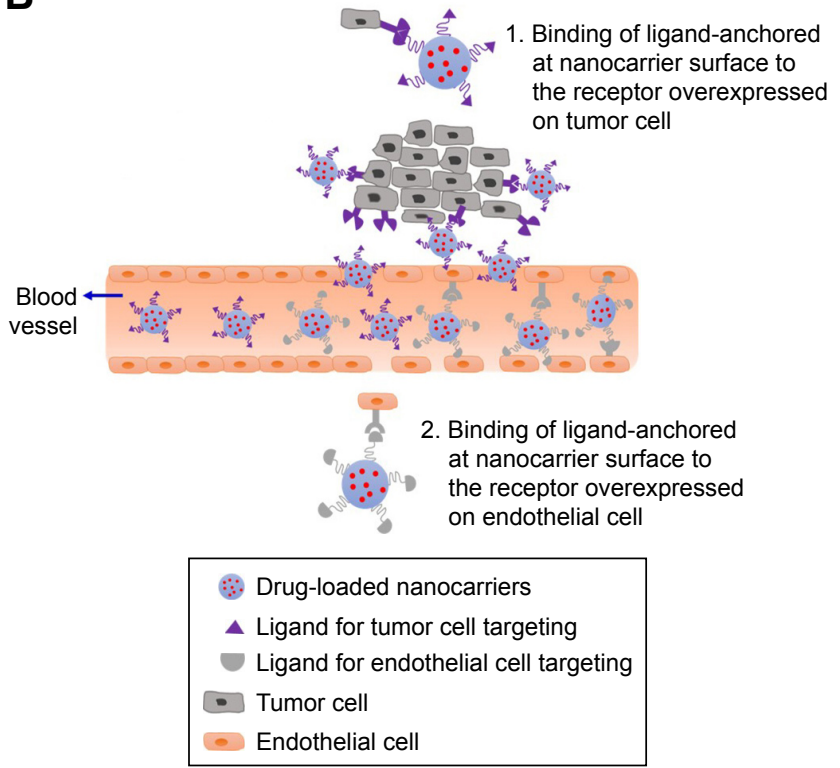

Figure 9 Diagrammatic illustration of passive tumor targeting $(\mathbf{A})$ and active tumor targeting $(\mathbf{B})$ by nanocarriers. Abbreviation: EPR, enhanced permeability and retention. 
tumor-targeting mechanism got approval for their clinical use. However, over or misinterpretation of the EPR effect, differences between the animal model and actual patients and abridged penetration of the nanomaterials into the targeted tissue and tumors are the most devastating drawbacks of passive tumor targeting that cannot be neglected. ${ }^{93,94}$

\section{Active mechanism}

Active targeting of specific tumor tissues is accomplished by surface modification of nanocarriers with site-specific targeting ligand (Figure 9B). The targeting ligands have the capability of binding to specific receptors exclusively overexpressed by tumor cells or tumor vasculature. ${ }^{95}$ The targeting agents commonly used to increase the site specificity of nanocarriers include small molecules, antibodies and antibody fragments, peptides (arginylglycylaspartic acid [RGD]), glycoproteins (transferrin), vitamins (folic acid), growth factors and nucleic acids. ${ }^{4,37}$ A high surface area to volume ratio of nanocarriers allows them to effectively attach multiple targeting moieties, thereby achieving better targeting of specific tumor cell types. ${ }^{95}$ Active tumor targeting is utilized not only to reduce the off-target delivery of chemotherapeutic agents but also to avoid the drawbacks of passive tumor targeting and overcome the multiple drug resistance. ${ }^{96}$ For the effective utilization of active targeting strategy, the desired target receptor must be homogeneously expressed in all target cells and the selected targeting moiety must exclusively bind to a receptor overexpressed by tumor cells only.

Active targeting is designed either to direct the liganddecorated nanocarrier to target the tumor cells or tumor microenvironment/vasculature. ${ }^{19}$ In the field of active targeting, both of these cellular targets have gained considerable attention in the recent past to improve the efficacy of chemotherapeutic agents and to reduce their adverse effects. Active targeting of tumor cells involves the targeting of overexpressed cell surface receptors with ligand-anchored nanocarrier via ligand-receptor interactions. ${ }^{97}$ The subsequent receptor-mediated endocytosis (internalization) improves the uptake of nanocarriers by cancer cells, thereby increasing intracellular drug concentration with no or only little increase in tumor accumulation..$^{98}$ Most commonly targeted cell surface receptors overexpressed on tumor cells in many types of tumors include folate receptor (FR), TfR, epidermal growth factor receptor (EGFR) and cell surface glycoproteins. ${ }^{19,95}$ Specific tumor type may have one of these tumor markers overexpressed on its cells surface. For instance, FR is overexpressed in breast, lung, ovarian and colorectal cancer cells; therefore, these cancer cells can be target with folate-modified nanocarriers. ${ }^{99}$ Likewise, the surface of endothelial cells of the blood-brain barrier has highly expressed TfRs, and therefore, transferrin can be utilized as a ligand for targeted delivery of antitumor drugs into the brain. ${ }^{100}$ Actively targeting the tumor vasculature (tumor endothelium) instead of tumor cells in an alternative strategy offers many advantages over the aforementioned one. Targeting the tumor vasculature involves the destruction of endothelial cells of tumor vessel, thereby blocking oxygen and nutrient supply of tumor cells with the ultimate result of tumor cells' death. ${ }^{101}$ Active tumor vascular targeting with ligand-attached nanocarriers might be achieved by targeting a number of moieties on the vascular wall, including vascular endothelial growth factors (VEGFs), vascular cell adhesion molecule (VCAMs), matrix metalloproteases (MMPs) and $\alpha_{\mathrm{v}} \beta_{3}$ integrins. ${ }^{101,102} \mathrm{~A}$ few representative examples of active tumor targeting using various nanocarriers surface modified with site-specific ligands are described here. In a study, Choi and Park ${ }^{103}$ reported the development of docetaxel-loaded nanocrystals having surface modification with transferrin to endow them with active tumor-targeting function. Transferrin-modified docetaxel nanocrystals exhibited higher cellular uptake and cytotoxicity in human lung A549 cancer cell line as compared to docetaxel nanocrystals and pure drug. In another study, apatinib-loaded liposomes were surface modified with targeting ligand, cyclic arginylglycylaspartic acid (cRGD), to specifically recognize $\alpha v \beta 3$ integrin. ${ }^{104}$ These cRGDanchored liposomes, compared with nontargeted liposomes, showed improved cellular uptake and inhibition of tumor growth in human colon HCT116 cancer cell line. Similarly, $\mathrm{Wu}$ et $\mathrm{al}^{105}$ investigated the effect of folate targeting on the antitumor efficacy of paclitaxel-encapsulated PMs in human esophageal cancer cell lines, EC9706. It was demonstrated that the folate-modified paclitaxel-loaded micelles were more effective in inhibiting tumor growth than free drug solution and plain paclitaxel micelles, when administered intravenously to tumor-bearing nude mice at an equivalent dose of $20 \mathrm{mg} / \mathrm{kg}$.

\section{Selected tumors and relative nanocarriers}

Tumor is mainly caused in all cases by mutation or any other defect either in the proto-oncogenes responsible for regulating cell proliferation and differentiation or in the tumor suppressor genes responsible for inhibiting cell growth and stimulating programmed cell death (apoptosis). ${ }^{37}$ These mutated genes are responsible for development of cancerous cells with unique features of uncontrolled cell growth, 
inability to switch off the excessive cell division, absence of apoptosis and ability to invade the adjacent and distant tissues. ${ }^{106}$ The risk factors for genetic mutations include radiation; chemical substances, ie, carcinogens; physical irritants; heredity and viruses. ${ }^{107}$ Tumors are one of the most devastating diseases worldwide, increasing with the rate of $>10$ million new cases per annum. ${ }^{108} \mathrm{~A}$ report of cancer research in the UK showed that the lifetime risk of cancer was $25 \%$ in 1975 , which was increased to $45 \%$ till 2009 , and they concluded that it will reach to $50 \%$ by $2027 .{ }^{109}$ However, the mortality rate of cancer has now declined in the past few years due to improved understanding of tumor biology and enhanced diagnostic procedures and treatments. ${ }^{110}$

Conventional chemotherapies adapted for the tumor management have many toxic side effects, including hepatic, renal, bone marrow, pulmonary, gastrointestinal and cardiac toxicities. ${ }^{111}$ Researchers are trying to limit the dose of chemotherapeutic drugs by targeting the specific tumor cells without exposing the normal cells to the toxic effects of drug. ${ }^{112}$ One of the landmarks in this regard is the development of nanocarriers that hold a great promise to enhance the therapeutic effectiveness and safety profile of conventional chemotherapeutic agents. These drug-loaded nanocarriers are capable of delivering antitumor chemotherapeutics to the tumor sites either by exploiting the pathophysiology of tumors or by decorating them with site-specific ligands. ${ }^{6}$ Four distinct types of tumors including breast, pancreatic, colorectal and lung tumors are selected and described in this review due to their worldwide prevalence and their high mortality rates, and the applications of relative nanocarriers in these tumors are discussed (Table 1).

\section{Breast tumor}

Breast tumor is the most commonly diagnosed tumor with the highest number of tumor deaths among females globally. Based on the GLOBOCAN report, $\sim 1.7$ million fresh breast tumor incidents and 522,000 demises occurred in 2012 worldwide. ${ }^{108}$ Even though the modern treatments frequently offer exceptional immediate prognoses, $\sim 13 \%$ of the patients develop locoregional recurrence within 9 years of primary treatment, out of which $25 \%$ patients have distant metastatic syndrome at the time of recurrence. ${ }^{113,114}$ Moreover, $>60 \%$ of the patients with localized breast tumor have distant, advanced stage tumor. ${ }^{115}$ For such patients, standard treatment comprises neoadjuvant chemotherapy trailed by surgical resection, radiation and additional adjuvant chemotherapy. One of the many aims of the neoadjuvant chemotherapy is to reduce locoregional tumor weight and size to reduce surgical process, permitting breast protection in various cases. Furthermore, neoadjuvant treatment can prevent additional metastatic spread of the disease.

Various chemotherapeutic agents are used to treat breast tumor in both neoadjuvant and adjuvant management procedures, including doxorubicin, paclitaxel and cisplatin. Doxorubicin is the first-line agent in breast tumor treatment. It reduces growth of tumor cells by preventing deoxyribonucleic acid (DNA) intercalation and macromolecular biosynthesis inside tumorous cells. Major problems linked with doxorubicin are hair loss, increased neutrophils and cardiac problems, which can be attributed to its toxicity associated with the increasing dose. Platinum agents that include cisplatin or carboplatin along with other combinational drugs are usually used as antitumor drugs for the management of breast tumor. Cisplatin triggers apoptosis by upholding DNA binding and cross-linking, thus preventing growth of tumor cells. The most distinguished toxicities associated with this drug include its neurotoxicity, nephrotoxicity and ototoxicity, which are related to high peak plasma concentration levels. ${ }^{116}$ As a matter of fact, these drugs might be rationally efficient in the adjuvant setting, but their modest toxicity profiles need to be improved to ensure their safety, efficacy and tolerability in combination therapies. For this purpose, various antitumor drug-loaded nanocarriers were used for their chemotherapeutic action as novel techniques for the targeted drug delivery through the locoregional lymphatics, leading to improved drug delivery and targeting tumorous cells with the lowest systemic toxicity while retaining therapeutic systemic levels. ${ }^{117}$

Tamoxifen-loaded biodegradable poly(o-caprolactone) nanocarriers were developed for the treatment of estrogen receptor-positive breast tumor. ${ }^{118}$ This study suggested that the NP preparations of selective estrogen receptor modulators, such as tamoxifen, would increase their therapeutic efficacy by delivering drug in the area of the estrogen receptor. These tamoxifen-loaded NPs were found to have a mean particle size of $<300 \mathrm{~nm}$ and a narrow size distribution. Similarly, a pH-responsive biodegradable system holding poly(ethylene oxide) (PEO)-modified poly( $\beta$-amino ester) NPs were developed by Potineni et $\mathrm{al}^{119}$ for the delivery of paclitaxel as an antitumor agent against breast tumor. Controlled solvent-displacement method was used to develop the paclitaxel-loaded nanocarriers. This study demonstrated that the PEO-modified poly $(\beta$-amino ester) NPs were in the nanosize range of 100-150 nm with a faster degradation profile in association with other synthetic polyesters. Moreover, $97 \%$ of paclitaxel-loading efficiency was 
Table I Antitumor drug-loaded nanocarriers for the treatment of various tumors

\begin{tabular}{|c|c|c|c|c|}
\hline Nanocarrier & Drug(s) & Tumor & Benefits & References \\
\hline SLNs & $\begin{array}{l}\text { 5-FU, doxorubicin, } \\
\text { paclitaxel, } \\
\text { methotrexate }\end{array}$ & $\begin{array}{l}\text { Colon, breast, } \\
\text { lungs, pancreatic }\end{array}$ & $\begin{array}{l}\text { a) SLN formulations have been successfully } \\
\text { prepared using a simple double emulsion } \\
\text { procedure that offers a better flexibility and least } \\
\text { process-related stress on the encapsulated drug. } \\
\text { These formulae represent a platform for the } \\
\text { preparation of SLNs for water-soluble anticancer } \\
\text { drugs, including peptides } \\
\text { b) They have shown higher cytotoxicity than the } \\
\text { equivalent amount of free-drug treatment as a } \\
\text { result of the synergetic effect }\end{array}$ & $|4-17| 59-16 \mid$, \\
\hline Liposomes & $\begin{array}{l}\text { Doxorubicin, cisplatin, } \\
\text { Doxil }\end{array}$ & Breast, lungs, colon & $\begin{array}{l}\text { a) The therapeutic advantages of targeted liposomes } \\
\text { compared with their nontargeting counterparts in } \\
\text { cancer cells have been demonstrated } \\
\text { b) Enhanced drug entrapment, leading to substantial } \\
\text { anticancer efficacy and abridged cardiotoxicity }\end{array}$ & $122,162,163$ \\
\hline Dendrimers & $\begin{array}{l}\text { Methotrexate, 5-FU, } \\
\text { cisplatin, doxorubicin }\end{array}$ & Breast, skin, lungs & $\begin{array}{l}\text { a) Internalization of the drug conjugates into the } \\
\text { tumor cells, resulting in increased antitumor } \\
\text { activity and reduced toxicity } \\
\text { b) These conjugates were found to have } \\
\text { favorable controlled release characteristic for } \\
\text { anticancer drugs }\end{array}$ & $25,26,28,29,164,165$ \\
\hline PNPs & $\begin{array}{l}\text { Doxorubicin, docetaxel, } \\
\text { paclitaxel, cisplatin, } \\
\text { imatinib mesylate }\end{array}$ & $\begin{array}{l}\text { Breast, chronic } \\
\text { myeloid leukemia }\end{array}$ & $\begin{array}{l}\text { a) A single intravenous injection of doxorubicin } \\
\text { conjugated to PLGA NP exhibited tumor } \\
\text { suppression comparable to that by daily injection } \\
\text { of free doxorubicin over I } 2 \text { days; thus, the NP } \\
\text { formulation was much more potent and longer } \\
\text { lasting than conventional free doxorubicin } \\
\text { b) Much greater cytotoxic potency to cancer cells } \\
\text { than Taxotere (current clinical form of docetaxel) } \\
\text { c) Paclitaxel-loaded PEG-PLGA-based NPs exhibited } \\
\text { enhanced in vitro and in vivo cytotoxic effects } \\
\text { compared with the commercial formulation of } \\
\text { paclitaxel (Taxol) } \\
\text { d) Cisplatin-loaded glycol chitosan NPs showed } \\
\text { sustained cisplatin release, improved antitumor } \\
\text { efficacy and decreased toxicity as compared to } \\
\text { free drug }\end{array}$ & $3 \mathrm{I}, 35-38,40,4 \mathrm{I}$ \\
\hline PMs & $\begin{array}{l}\text { Methotrexate, cisplatin, } \\
\text { paclitaxel, docetaxel, } \\
\text { doxorubicin }\end{array}$ & Breast, skin, lungs & $\begin{array}{l}\text { a) PMs increase the anticancer drug circulation time } \\
\text { in the blood } \\
\text { b) The smaller size (10-100 nm) and prolonged } \\
\text { circulation times in vivo cause the PMs to } \\
\text { preferentially accumulate in the tumor site and } \\
\text { increase their cytotoxic effect }\end{array}$ & $39,43,46-48,166,167$ \\
\hline CNTs & $\begin{array}{l}\text { Methotrexate, cisplatin, } \\
\text { paclitaxel, doxorubicin, } \\
\text { tripiscian, carboplatin, } \\
\text { mitomycin C }\end{array}$ & Lungs, breast, skin & $\begin{array}{l}\text { a) The needle-like shape of CNTs allows them } \\
\text { to cross the cell membrane via endocytosis or } \\
\text { "needle-like penetration" and subsequently enter } \\
\text { into the cell } \\
\text { b) They offer unique physicochemical } \\
\text { characteristics, great drug entrapment, intrinsic } \\
\text { stability, mechanical flexibility and suitable surface } \\
\text { functionalization }\end{array}$ & $68-76,168-174$ \\
\hline VNPs & $\begin{array}{l}\text { Doxorubicin, paclitaxel, } \\
\text { methotrexate }\end{array}$ & Breast, colon, lungs & $\begin{array}{l}\text { a) VNPs show numerous striking characters } \\
\text { comprising biocompatibility, morphological } \\
\text { consistency, easy surface functionalization and } \\
\text { availability in a variety of sizes and shapes } \\
\text { b) PEGylating the surface of VNPs can increase their } \\
\text { circulation time in the host }\end{array}$ & $53,54,57,58,175$ \\
\hline
\end{tabular}

Abbreviations: 5-FU, 5-fluorouracil; CNT, carbon nanotube; NP, nanoparticle; PEG-PLGA, PEGylated poly(lactic-co-glycolic acid); PLGA, poly(lactic-co-glycolic acid); PM, polymeric micelle; PNP, polymeric nanoparticle; SLN, solid lipid nanoparticle; VNP, virus-based nanoparticle. 
found at $1.0 \%(\mathrm{w} / \mathrm{w})$ drug concentration. They concluded that the $\mathrm{pH}$-sensitive solubility of the drug-loaded nanocarriers would help in drug release at the core of solid tumors, leading to enhanced therapeutic effects by distributing the encapsulated drug to solid tumors. ${ }^{119}$

In another study, hydrophobic anticancer drug was successfully loaded to biodegradable PNPs by physical adsorption of triblock polymer. ${ }^{120}$ The authors of this study managed to attain specific tumor targeting and circulating of drug reservoir for tamoxifen, a drug of choice in the treatment of breast tumor. Tamoxifen-loaded PEO-modified $\operatorname{poly}(\alpha$-caprolactone) nanocarriers were prepared by the solvent-displacement method with a particle size of $\sim 200 \mathrm{~nm}$. These antitumor drug-loaded nanocarriers showed tumor selective biodistribution with clinically consumable circulation time.

One of the most prominent clinical hindrances today is the multidrug-resistant (MDR) characteristics that may result in cancer nonresponsive behavior and sometimes its recurrence. Nanotechnology-based chemotherapy was used to treat these clinical situations. For this purpose, Milane et al ${ }^{121}$ developed EGFR-targeted polymer blend nanocarriers loaded with paclitaxel and lonidamine to treat human breast cancer. Their results demonstrated that the polymeric nanocarriers with characteristics of efficient drug loading and sustained drug release resulted in improved drug combination therapy with proficient EGFR targeting. The electron spectroscopy chemical analysis (ESCA) study was used to determine the fact that the nanocarrier surfaces were having the targeting sites/paradigm. These results were further confirmed by targeting these nanocarriers in the cell lines, which in response resulted in an active EGFR targeting. Thus, the combination of $1 \mu \mathrm{M}$ paclitaxel with $10 \mu \mathrm{M}$ lonidamine improved $5 \%-10 \%$ cell viability as compared to the treatment with $1 \mu \mathrm{M}$ paclitaxel alone in the MDR cells.

Nanoconjugation-based combined drug delivery of doxorubicin and cisplatin for the treatment of locally advanced breast tumor with enhanced efficiency and reduced toxicity was another successful antitumor drug combination study performed by Cohen et al. ${ }^{122}$ Doxorubicin-cisplatin-loaded nanocarriers were administered subcutaneously, and their therapeutic effect was achieved as a result of the cleavage of hyaluronan in the lymph, leading to the transport of free drug to targeted sites through active or diffusion mechanism. This antitumor drug combination showed considerably enhanced efficacy and reduced toxicity as compared to the standard agent at all experimental doses, leading to comprehensive pathologic tumor response.
An emergent approach for tumor treatment is the effective use of nanocarrier-based combination drug delivery systems. Such systems, when administered in sustained, targeted and controlled drug delivery form, have not only overcome some biological and biomedical hurdles but at the same time have led to increased therapeutic effects with reduced cytotoxic possessions.

\section{Pancreatic tumor}

Pancreatic tumor is believed to be the fourth leading reason of cancer deaths in both genders, with a projected 53,670 newly diagnosed pancreatic tumor cases and 43,090 deaths in the US in $2017 .{ }^{123}$ If diagnosed well in time, only $10 \%$ cases of the pancreatic tumor patients could be cured through surgical procedures. ${ }^{124}$ In some cases of surgically resectable tumor, aggressive metastasis resurgence occurs that promotes resistance to conventional chemotherapy and radiation therapy. Instead of the significant improvement in cancer finding, surgical removal, chemotherapy and radiation therapy, diagnosis of the disease remained deprived with a survival rate of $5 \%$ per 5 years. Poor management is chiefly because of the diagnosis of disease at advanced stages of localized tumor or metastatic tumor growth. Patients with an advanced stage localized pancreatic tumor demonstrate an average of 6-10 months survival, whereas those with a metastatic form of the disease only have a 3-6 months median survival. ${ }^{125}$

Chemotherapy is still widely used for the treatment of localized advanced stage pancreatic tumor or metastatic pancreatic adenocarcinoma. For example, gemcitabine $\left(2^{\prime}-2^{\prime}-\right.$ difluorodeoxycytidine) is used as a frontline therapeutic drug for pancreatic tumor. However, gemcitabine when combined with other therapeutic mediators, such as platinum analogs, ${ }^{126}$ antimetabolites ${ }^{127}$ or topoisomerase inhibitors, ${ }^{93}$ did not improve the therapeutic outcome or survival rate. FOLFIRINOX replaced gemcitabine-based therapy owing to its better average overall survival of 11.1 months in comparison to 6.8 months of the former. ${ }^{128}$ Nevertheless, an optimal therapeutic regimen for pancreatic tumor is not yet obtained, as a number of chemotherapeutic clinical trials were declared unsuccessful in their Phase II or III stage due to lake of remarkable results. A recent study demonstrated that none of the FOLFIRINOX and gemcitabine-based regimen proved a better outcome against advanced or metastatic pancreatic tumor in their clinical trials. ${ }^{129}$ Thus, it is important to reconsider the current therapeutic approaches and develop novel schemes for the management of pancreatic tumor.

Nanotechnology is extensively utilized for the development of particles of nanosize range from biomedically important 
organic or inorganic resources. The recent improvements in nanotechnology proved better results for tumor diagnostics, imaging and site specific delivery. ${ }^{130}$ Nanocarriers are also used for increasing the dose-dependent efficacy of therapeutic or imaging contrast agents by enhancing their bioavailability. Similarly, they are used for selected targeting of tumor cells to upsurge image resolution and/or decrease the toxic effects of the chemotherapeutic agents. ${ }^{131}$ These nanocarriers are used for treating metastatic tumors such as pancreatic and lung tumors. However, for preparing effective nanocarriers both as imaging contrast agents or therapeutics mediators, complete understanding of the physiological barriers associated with the disease is essential. This knowledge would help to understand the mechanism and use of nanocarriers, particularly the NP release, accretion and infiltration into the tumor milieu. ${ }^{93}$

Nanocarriers such as liposomes and polymeric nanomaterials were used to encapsulate, target and deliver a wide variety of chemotherapeutic agents. Doxil, the first NP drug approved by the US Food and Drug Administration (FDA) in 1995, was actually liposomal formulation having a diameter of $100 \mathrm{~nm}$ encapsulating an antitumor drug, doxorubicin. This encapsulation of doxorubicin in liposomes largely changed the pharmacokinetic and pharmacodynamics characteristics of the drug, which in turn led to greater uptake of tumor and resulted in an enhanced activity of antitumor drug. ${ }^{125}$ Nowadays, Doxil is used for the treatment of a wide range of solid tumors and platinum-resistant ovarian cancer. ${ }^{128}$ Chemotherapeutic drug oxaliplatin-loaded nanoparticulate micelles were prepared by Cabral et al, ${ }^{126}$ with slow release of encapsulated drug once they were in contact with the tumor microenvironment, resulting in enhanced antitumor effect. Similarly, encapsulated nanocarriers of gemcitabine were used as the first-line treatment for the locally advanced or metastatic pancreatic tumor. ${ }^{132}$ Pancreatic tumor was also treated with albumin-bound paclitaxel-loaded nanocarriers, also called as nab-paclitaxel. ${ }^{133}$ Iron oxide nanoparticles (IONPs) were also prepared for expressing a uPAR-targeted moiety on their surface. ${ }^{129}$ Nanocarriers were also prepared in the form of liposomes, lipid polymers and dendrimers, which act as a vehicle for the delivery of siRNAs. ${ }^{134,135}$ These NPs were designed in such a way that they can assemble themselves with siRNA, leading to its protection from degradation and rapid clearance from the body. Furthermore, these NPs have the capability to retain multifunctional constituents for targeted siRNA delivery and proficient infiltration into targeted cell. ${ }^{136}$ Similarly, nanoparticulate liposomes, consisting of a superficially attached TfR antibody with siRNA against HER-2, were used in the treatment of pancreatic tumor. ${ }^{137}$ It was observed in a murine xenograft model that TfR-targeting nanocarriers improved the distribution of siRNA into pancreatic tumor milieu in comparison to the nanocarriers without TfR antibody. Additionally, site-specific provision of the NP-siRNA complexes not only were capable of quieting HER-2 expression in the pancreatic tumor cells but also proliferated sensitivity to gemcitabine.

\section{Colorectal tumor}

Colorectal tumor is the third most frequently diagnosed tumor in males, accounting for a total 1.4 million new cases and 693,900 deaths in 2012 worldwide. ${ }^{108}$ The risk factors associated with colon tumor are both from environment and geography. Environmental factors presumably modulate the genetic mutation, while geographical location induces the colorectal tumor among various countries of the world. Mucosal colonic polyps result in colon cancer, which have two most common histological types, including hyperplastic and adenomatous polyps. Hyperplastic polyps consist of an enhanced number of glandular cells but reduced cytoplasmic mucus. They lack nuclear hyperchromatism and atypia. ${ }^{138}$ Similarly, the adenomatous polyps are typically cigar structured, hyperchromatic, inflated and packed in palisade shape. A literature study shows that all the tumors result from adenomas as described by their clinical, pathological, histological and epidemiological demonstrations. ${ }^{138}$ Colon tumor is more probable to develop if the patient has increased number of adenomas polyps. This case gets even more severe if it is familial adenomatous polyps and if colectomy is not done. The colorectal patients may be resected in spite of the manifestation of distant metastasis to avoid intestinal hindrance and hemorrhage followed by systemic chemotherapy for the treatment of metastases. However, many institutions prefer systemic chemotherapy as the first treatment followed by resection for those patients with symptomatic disease. This could be attributed to the postoperative complications, which may result in discontinuation of chemotherapy.

Numerous treatment strategies are currently in use to fight against this tumorous condition, including surgical procedure, radiation therapy, radiofrequency ablation, targeted rehabilitation, cryosurgery and chemotherapy. The most prominent and appropriate of all above is the chemotherapy treatment, which has improved the patients' compliance and their quality of life. ${ }^{139}$ Although it is considered a quality treatment approach, yet, conventional chemotherapy results in less concentration of drug to be bioavailable at the site of tumor, making it less effective approach for tumor treatment. 
In the recent advanced era of nanotechnology, various nanocarriers were successfully used to obtain the desired effects of targeted treatment against colorectal tumor. These nanocarriers were used to carry the approved colorectal anticancer drugs including 5-FU, irinotecan, oxaliplatin, bevacizumab and capecitabine. ${ }^{140}$ One of such studies was conducted by Yassin et al in 2010. They prepared 5-FU solid lipid nanocarriers using the double emulsion-solvent evaporation technique. The SLNs were having nanosize (402 nm) with high entrapment efficiency of $\sim 51 \%$. They showed extended release profile of $60 \%$ in 8 hours in the simulated colonic medium. In another study, oxaliplatinloaded PLAGA microspheres were developed with remarkable entrapment efficiency (up to 90\%). ${ }^{141}$ These microspheres showed diverse release profiles, including (type II) zero-order kinetics and the orthodox sigmoid release profile with different burst release and plateau sizes. These findings improved the efficiency of local tumor treatment.

5-FU-loaded hyaluronic acid (HA)-conjugated silica nanocarriers were prepared by Liu et $\mathrm{al}^{142}$ to treat colon tumor. They demonstrated the attachment and intracellular accumulation of 5-FU-loaded nanocarriers on the basis of their HA surface alterations in colon tumorous cells. The nanocarriers were having a size of $\sim 130 \mathrm{~nm}$ with spherical shapes. They showed sustained release of their incorporated drug over a period of 120 hours. 5-FU-loaded HA-conjugated nanocarriers resulted in an enhanced antitumor efficacy and decreased side effects as compared to the nontargeted nanocarriers. Docetaxel-loaded biodegradable dendrimers were prepared by Lee et $\mathrm{al}^{28}$ with multiple attachment sites, controlled drug loading and release. These drug-loaded dendrimers were 10 times less toxic than free docetaxel in the treatment of colon tumor. They also showed $100 \%$ antitumor efficacy after a treatment of 60 days.

\section{Lung tumor}

Lungs tumor was estimated as the most commonly detected tumor and the leading reason of cancer deaths among males, with 1.8 million total new cases and 1.5 million deaths occurring in 2012 globally. ${ }^{108}$ Metastasis of lung tumor cells to a secondary location such as breast and vice versa accounts for the vast majority of tumor deaths and presents a major challenge in cancer treatment. ${ }^{143}$ One of the main problems of existing chemotherapy for lung tumor is its inadequate efficiency and specificity. Thus, it is always required to develop site-specific and targeted therapies to attain adequate efficacy and reduced side effects. The recent advancement in nanomedicine technology has attracted a great attention as the antitumor medicine of next generation based on integrated imaging and therapeutic responses (eg, image-visible nanotherapeutics).

A few of the problems related with the conservative diagnostic methods of lungs tumor were their expensive procedures and inaccuracy, which make them inappropriate choices for tumor screening. ${ }^{144}$ For this purpose, sensorbased gold NPs were successfully developed and used to diagnose the lung tumor. They were used to differentiate the breath of lungs tumor patients from those of healthy people in a highly humid atmosphere. These sensor-based NPs were cheap and noninvasive diagnostic devices for lung tumor. ${ }^{145}$ Similarly, PLGA-based anticancer drug (TAS-103)loaded NPs were prepared with an average particle size of $200 \mathrm{~nm}$, which resulted in enhanced antitumor efficacy in lungs tumor. ${ }^{146}$ Moreover, doxorubicin contained in poly(butylcyanoacrylate) NPs were also effective against lung tumor. ${ }^{147}$ Liposomes were also used as nanocarriers for various drugs in the targeting of lung tumor. ${ }^{18}$ A multicomponent liposomal formulation encapsulating two cytotoxic agents (etoposide and docetaxel) was prepared for p53 tumor suppressor gene-mediated enhanced cytotoxicity in A549 and H-1299 lung tumor cell lines. ${ }^{148}$ Likewise, the liposomal formulation of 9-nitrocamptothecin through nebulizers was very effective against metastatic lung tumors both in vivo and in vitro. ${ }^{149}$ Furthermore, interleukin (IL)-2 liposomes was administered to patients through inhalation with great effectiveness against pulmonary metastases.

Gelatin-based nanoparticles (GNPs) were also used for the delivery of hydrophilic and hydrophobic antitumor drugs such as paclitaxel, ${ }^{150}$ cisplatin, ${ }^{151}$ curcumin, ${ }^{152}$ resveratrol ${ }^{153}$ and methotrexate. ${ }^{154}$ GNPs also showed greater antitumor activity, sustained drug release and exerted very low toxicity to cells. Recently, doxorubicin-loaded NPs equipped with effervescent molecules as excipient were developed. ${ }^{30}$ These effervescent NPs consisting of doxorubicin gained deep lung deposition and were scattered primarily in the lung and had negligible deposition in other tissues or organs. Additionally, Taratula et $\mathrm{al}^{155}$ used MSNs as a carrier for inhalation therapy with specific targeting to lungs tumor. This formulation consisted of five chief components, namely, MSN, loaded drugs (cisplatin and doxorubicin), pump (MRP1) and non-pump suppressors (BCL2) of drug resistance and particular targeting ligand (LHRH peptide) for lung tumor. In this experiment, the immediate delivery of chemotherapeutic drugs and suppressor proteins via inhalation to A549-bearing NCR nude mice resulted in higher accumulation of nanocarriers in the lungs. In addition, the delivery route preserved the activities 
of both chemotherapeutic drugs and siRNA, which in turn resulted in enhanced apoptotic actions in lung cells. ${ }^{155,156}$

Nevertheless, COX-2 inhibitor, celecoxib, was encapsulated into nanostructured lipid carriers by using triglycerides such as Compritol and Miglyol, through high pressure homogenization method with reduced particle size and enhanced antitumor efficacy. ${ }^{157}$ Similarly, 5-FU-loaded, lipid-coated NPs were developed, and their in vivo pharmacokinetics was studied in hamsters after inhalation delivery. It was observed that these lipid-coated NPs exhibited sustained 5-FU delivery and efficient local targeting in the lung tumor sites. ${ }^{158}$

\section{Future perspective}

One of the main challenges of recent advancement in nanotechnology to be effectively used for the treatment of various tumors is the expansion of new-generation nanocarrier drugs. This expansion would certify the vibrant targeting of tumors by interacting with the surface-attached ligand and the receptors on the selected cells and tissues. However, it requires some hurdles to overcome such as a lack of sufficient expertise, difficulty in crossing the cell membrane, narrow therapeutic window of drugs, regulatory hurdles and costeffectiveness.

Unfortunately, the typical recurrence of formulationdriven expansion has not attained the projected patient compliance; even then, nanocarriers have the capability to achieve the target set for cancer therapeutics, both in case of the conventional and next-generation agents. Different targeted nanocarriers have established an enhanced therapeutic activity in numerous animal models of tumors. More precisely, $\sim 120$ ongoing clinical trials with numerous antibody containing nanocarrier formulations are under investigations. Similarly, today the scientists are able to image the type and location of the tumor, which in turn lead to paradigm the appropriate therapeutic regimens. Moreover, if the tumor cells are of circulating type as it happened in lymphoma and leukemia, a carrier with long circulating half-life and high capability of targeting surface antigen are preferred. It is also hoped in the near future that the scientists would be able to develop targeted molecular composites that may lead to enhanced therapeutic results with abridged expenses.

Although researchers have investigated and developed a number of new drug delivery systems to achieve drug efficiency in patients, yet, only a few of these auspicious preclinical drug delivery systems have reached the market. This could be attributed to the vital gaps in the transformation of anticancer drug-loaded nanocarriers. Thus, it is important to modify some of the traditional models to circumvent these problems. In this respect, highly sophisticated efforts are required to resolve some issues on urgent basis to accomplish innocuous usage of the newly developed nanocarriers in the clinical studies. These include the development of standard nanoformulations validated through in vitro and in vivo assay for efficacy, safety and potential toxicities.

\section{Conclusion}

Nanotechnology has recently been developed as one of the latest approaches for tumor drug delivery. We discussed numerous nanocarriers that are used as emerging tools for the treatment of various tumors. These nanocarriers have brought a revolution in cancer drug delivery by targeting tumor explicitly with enriched permeability and withholding effect as much as required. These exciting progressions in cancer therapy and exclusive development of numerous novel drug delivery systems have augmented the hope of those fighting against tumors. It is believed that in future, management of precise dose of drug with the highest systemic release from the nanocarriers and minimum toxic effects will not only enhance the use of nanocarrier systems for antitumor drug delivery but also improve the patient compliance.

\section{Acknowledgment}

This work was partially supported by the Higher Education Commission of Pakistan through a grant (No 21-836/SRGP/ $\mathrm{R} \& \mathrm{D} / \mathrm{HEC} / 2016)$.

\section{Disclosure}

The authors report no conflicts of interest in this work.

\section{References}

1. Neubert RHH. Potentials of new nanocarriers for dermal and transdermal drug delivery. Eur J Pharm Biopharm. 2011;77(1):1-2.

2. Mishra B, Patel BB, Tiwari S. Colloidal nanocarriers: a review on formulation technology, types and applications toward targeted drug delivery. Nanomedicine. 2010;6(1):9-24.

3. How CW, Rasedee A, Manickam S, Rosli R. Tamoxifen-loaded nanostructured lipid carrier as a drug delivery system: characterization, stability assessment and cytotoxicity. Colloids Surf B Biointerfaces. 2013;112: 393-399.

4. Sun T, Zhang YS, Pang B, Hyun DC, Yang M, Xia Y. Engineered nanoparticles for drug delivery in cancer therapy. Angew Chem Int Ed. 2014; 53(46):12320-12364.

5. Wong HL, Bendayan R, Rauth AM, Li Y, Wu XY. Chemotherapy with anticancer drugs encapsulated in solid lipid nanoparticles. Adv Drug Deliv Rev. 2007;59(6):491-504.

6. Wang AZ, Langer R, Farokhzad OC. Nanoparticle delivery of cancer drugs. Annu Rev Med. 2012;63:185-198.

7. Müller RH, Mäder K, Gohla S. Solid lipid nanoparticles (SLN) for controlled drug delivery - a review of the state of the art. Eur J Pharm Biopharm. 2000;50(1):161-177.

8. Malam Y, Loizidou M, Seifalian AM. Liposomes and nanoparticles: nanosized vehicles for drug delivery in cancer. Trends Pharmacol Sci. 2009;30(11):592-599. 
9. Üner M, Yener G. Importance of solid lipid nanoparticles (SLN) in various administration routes and future perspectives. Int J Nanomedicine. 2007;2(3):289-300.

10. Mehnert W, Mäder K. Solid lipid nanoparticles: production, characterization and applications. Adv Drug Deliv Rev. 2001;47(2-3):165-196.

11. Zeb A, Qureshi OS, Kim HS, et al. High payload itraconazoleincorporated lipid nanoparticles with modulated release property for oral and parenteral administration. J Pharm Pharmacol. 2017;69(8): 955-966.

12. Müller RH, Radtke M, Wissing SA. Solid lipid nanoparticles (SLN) and nanostructured lipid carriers (NLC) in cosmetic and dermatological preparations. Adv Drug Deliv Rev. 2002;54(suppl 1):S131-S155.

13. Qureshi OS, Kim HS, Zeb A, et al. Sustained release docetaxelincorporated lipid nanoparticles with improved pharmacokinetics for oral and parenteral administration. J Microencapsul. 2017;34(3): 250-261.

14. Battaglia L, Gallarate M, Peira E, et al. Solid lipid nanoparticles for potential doxorubicin delivery in glioblastoma treatment: preliminary in vitro studies. J Pharm Sci. 2014;103(7):2157-2165.

15. Yuan H, Miao J, Du Y-Z, You J, Hu F-Q, Zeng S. Cellular uptake of solid lipid nanoparticles and cytotoxicity of encapsulated paclitaxel in A549 cancer cells. Int J Pharm. 2008;348(1-2):137-145.

16. Kakkar D, Dumoga S, Kumar R, Chuttani K, Mishra AK. PEGylated solid lipid nanoparticles: design, methotrexate loading and biological evaluation in animal models. Med Chem Commun. 2015;6(8):1452-1463.

17. Patel MN, Lakkadwala S, Majrad MS, et al. Characterization and evaluation of 5-fluorouracil-loaded solid lipid nanoparticles prepared via a temperature-modulated solidification technique. AAPS PharmSciTech. 2014;15(6):1498-1508.

18. Lee W-H, Loo C-Y, Traini D, Young PM. Nano-and micro-based inhaled drug delivery systems for targeting alveolar macrophages. Expert Opin Drug Deliv. 2015;12(6):1009-1026.

19. Deshpande PP, Biswas S, Torchilin VP. Current trends in the use of liposomes for tumor targeting. Nanomedicine. 2013;8(9):1509-1528.

20. Torchilin VP. Recent advances with liposomes as pharmaceutical carriers. Nat Rev Drug Discov. 2005;4(2):145-160.

21. Kresge C, Leonowicz M, Roth W. Dendrimers and Dendrons. Concepts, Syntheses, Applications. Weinheim: VCH; 2001.

22. Basu S, Sandanaraj BS, Thayumanavan S. Molecular recognition in dendrimers. In: Mark HF, editor. Encyclopedia of Polymer Science and Technology. 4th ed. John Wiley \& Sons, Inc; 2004.

23. Stiriba SE, Frey H, Haag R. Dendritic polymers in biomedical applications: from potential to clinical use in diagnostics and therapy. Angew Chem Int Ed. 2002;41(8):1329-1334.

24. de Groot FM, Albrecht C, Koekkoek R, Beusker PH, Scheeren HW. "Cascade-release dendrimers" liberate all end groups upon a single triggering event in the dendritic core. Angew Chem Int Ed. 2003;42(37): 4490-4494.

25. Lai P-S, Lou P-J, Peng C-L, et al. Doxorubicin delivery by polyamidoamine dendrimer conjugation and photochemical internalization for cancer therapy. J Control Release. 2007;122(1):39-46.

26. Malik N, Evagorou EG, Duncan R. Dendrimer-platinate: a novel approach to cancer chemotherapy. Anticancer Drugs. 1999;10(8):767-776.

27. Zhuo RX, Du B, Lu ZR. In vitro release of 5-fluorouracil with cyclic core dendritic polymer. J Control Release. 1999;57(3):249-257.

28. Lee CC, Gillies ER, Fox ME, et al. A single dose of doxorubicinfunctionalized bow-tie dendrimer cures mice bearing C-26 colon carcinomas. Proc Natl Acad Sci U S A. 2006;103(45):16649-16654.

29. Bhadra D, Bhadra S, Jain S, Jain N. A PEGylated dendritic nanoparticulate carrier of fluorouracil. Int J Pharm. 2003;257(1):111-124.

30. Rao JP, Geckeler KE. Polymer nanoparticles: preparation techniques and size-control parameters. Prog Polym Sci. 2011;36(7):887-913.

31. Bamrungsap S, Zhao Z, Chen T, et al. Nanotechnology in therapeutics: a focus on nanoparticles as a drug delivery system. Nanomedicine. 2012;7(8):1253-1271.

32. Prabhu RH, Patravale VB, Joshi MD. Polymeric nanoparticles for targeted treatment in oncology: current insights. Int J Nanomedicine. 2015;10:1001-1018.
33. Wang X, Wang Y, Chen ZG, Shin DM. Advances of cancer therapy by nanotechnology. Cancer Res Treat. 2009;41(1):1-11.

34. Hu CM, Aryal S, Zhang L. Nanoparticle-assisted combination therapies for effective cancer treatment. Ther Deliv. 2010;1(2):323-334.

35. Alexis F, Pridgen EM, Langer R, Farokhzad OC. Nanoparticle technologies for cancer therapy. In: Schäfer-Korting M, editor. Drug Delivery. Berlin, Heidelberg: Springer Berlin Heidelberg; 2010:55-86.

36. Zhu Y, Liao L. Applications of nanoparticles for anticancer drug delivery: a review. J Nanosci Nanotechnol. 2015;15(7):4753-4773.

37. Pérez-Herrero E, Fernández-Medarde A. Advanced targeted therapies in cancer: drug nanocarriers, the future of chemotherapy. Eur J Pharm Biopharm. 2015;93:52-79.

38. Khuroo T, Verma D, Talegaonkar S, Padhi S, Panda AK, Iqbal Z. Topotecan-tamoxifen duple PLGA polymeric nanoparticles: investigation of in vitro, in vivo and cellular uptake potential. Int J Pharm. 2014; 473(1-2):384-394.

39. Wang H, Zhao Y, Wu Y, et al. Enhanced anti-tumor efficacy by co-delivery of doxorubicin and paclitaxel with amphiphilic methoxy PEG-PLGA copolymer nanoparticles. Biomaterials. 2011;32(32): $8281-8290$

40. Wang W, Chen S, Zhang L, et al. Poly(lactic acid)/chitosan hybrid nanoparticles for controlled release of anticancer drug. Mater Sci Eng C Mater Biol Appl. 2015;46:514-520.

41. Anitha A, Deepa N, Chennazhi KP, Lakshmanan V-K, Jayakumar R. Combinatorial anticancer effects of curcumin and 5-fluorouracil loaded thiolated chitosan nanoparticles towards colon cancer treatment. Biochim Biophys Acta. 2014;1840(9):2730-2743.

42. Zhao D, Liu C-J, Zhuo R-X, Cheng S-X. Alginate/CaCO3 hybrid nanoparticles for efficient codelivery of antitumor gene and drug. Mol Pharm. 2012;9(10):2887-2893.

43. Biswas S, Kumari P, Lakhani PM, Ghosh B. Recent advances in polymeric micelles for anti-cancer drug delivery. Eur J Pharm Sci. 2016;83: 184-202.

44. Gothwal A, Khan I, Gupta U. Polymeric micelles: recent advancements in the delivery of anticancer drugs. Pharm Res. 2016;33(1):18-39.

45. Nakanishi T, Fukushima S, Okamoto K, et al. Development of the polymer micelle carrier system for doxorubicin. J Control Release. 2001; 74(1-3):295-302.

46. Rapoport N. Physical stimuli-responsive polymeric micelles for anticancer drug delivery. Prog Polym Sci. 2007;32(8-9):962-990.

47. Ren J, Fang Z, Yao L, et al. A micelle-like structure of poloxamermethotrexate conjugates as nanocarrier for methotrexate delivery. Int J Pharm. 2015;487(1-2):177-186.

48. Li X, Yang Z, Yang K, et al. Self-assembled polymeric micellar nanoparticles as nanocarriers for poorly soluble anticancer drug ethaselen. Nanoscale Res Lett. 2009;4(12):1502-1511.

49. Hamaguchi T, Matsumura Y, Suzuki M, et al. NK105, a paclitaxelincorporating micellar nanoparticle formulation, can extend in vivo antitumour activity and reduce the neurotoxicity of paclitaxel. Br J Cancer. 2005;92(7):1240-1246.

50. Manchester M, Singh P. Virus-based nanoparticles (VNPs): platform technologies for diagnostic imaging. Adv Drug Deliv Rev. 2006;58(14): 1505-1522.

51. Singh P, Prasuhn D, Yeh RM, et al. Bio-distribution, toxicity and pathology of cowpea mosaic virus nanoparticles in vivo. J Control Release. 2007;120(1-2):41-50.

52. Pattenden LK, Middelberg AP, Niebert M, Lipin DI. Towards the preparative and large-scale precision manufacture of virus-like particles. Trends Biotechnol. 2005;23(10):523-529.

53. Ma Y, Nolte RJ, Cornelissen JJ. Virus-based nanocarriers for drug delivery. Adv Drug Deliv Rev. 2012;64(9):811-825.

54. Jabir NR, Tabrez S, Ashraf GM, Shakil S, Damanhouri GA, Kamal MA. Nanotechnology-based approaches in anticancer research. Int $J$ Nanomedicine. 2012;7:4391-4408.

55. Douglas T, Young M. Viruses: making friends with old foes. Science. 2006;312(5775):873-875

56. Chen Z. Small-molecule delivery by nanoparticles for anticancer therapy. Trends Mol Med. 2010;16(12):594-602. 
57. Cao J, Guenther RH, Sit TL, Opperman CH, Lommel SA, Willoughby JA. Loading and release mechanism of red clover necrotic mosaic virus derived plant viral nanoparticles for drug delivery of doxorubicin. Small. 2014;10(24):5126-5136.

58. Honarbakhsh S, Guenther RH, Willoughby JA, Lommel SA, Pourdeyhimi B. Polymeric systems incorporating plant viral nanoparticles for tailored release of therapeutics. Adv Healthc Mater. 2013;2(7): 1001-1007.

59. Iijima S. Helical microtubules of graphitic carbon. Nature. 1991; 354(6348):56-58.

60. Bianco A. Carbon nanotubes for the delivery of therapeutic molecules. Expert Opin Drug Deliv. 2004;1(1):57-65.

61. Madani SY, Naderi N, Dissanayake O, Tan A, Seifalian AM. A new era of cancer treatment: carbon nanotubes as drug delivery tools. Int $J$ Nanomedicine. 2011;6:2963-2979.

62. Yan Y, Chan-Park MB, Zhang Q. Advances in carbon-nanotube assembly. Small. 2007;3(1):24-42.

63. Ng CM, Loh H-S, Muthoosamy K, Sridewi N, Manickam S. Conjugation of insulin onto the sidewalls of single-walled carbon nanotubes through functionalization and diimide-activated amidation. Int J Nanomedicine. 2016;11:1607-1614

64. Vardharajula S, Ali SZ, Tiwari PM, et al. Functionalized carbon nanotubes: biomedical applications. Int J Nanomedicine. 2012;7: 5361-5374.

65. Iannazzo D, Piperno A, Pistone A, Grassi G, Galvagno S. Recent advances in carbon nanotubes as delivery systems for anticancer drugs. Curr Med Chem. 2013;20(11):1333-1354.

66. Ajima K, Murakami T, Mizoguchi Y, et al. Enhancement of in vivo anticancer effects of cisplatin by incorporation inside single-wall carbon nanohorns. ACS Nano. 2008;2(10):2057-2064.

67. Wu W, Li R, Bian X, et al. Covalently combining carbon nanotubes with anticancer agent: preparation and antitumor activity. ACS Nano. 2009;3(9):2740-2750.

68. Fabbro C, Ali-Boucetta H, Ros TD, Kostarelos K, Bianco A, Prato M. Targeting carbon nanotubes against cancer. Chem Commun. 2012; 48(33):3911-3926.

69. Das M, Datir SR, Singh RP, Jain S. Augmented anticancer activity of a targeted, intracellularly activatable, theranostic nanomedicine based on fluorescent and radiolabeled, methotrexate-folic Acid-multiwalled carbon nanotube conjugate. Mol Pharm. 2013;10(7):2543-2557.

70. Lay CL, Liu HQ, Tan HR, Liu Y. Delivery of paclitaxel by physically loading onto poly (ethylene glycol)(PEG)-graft carbon nanotubes for potent cancer therapeutics. Nanotechnology. 2010;21(6):065101.

71. Adeli M, Beyranvand S, Hamid M. Noncovalent interactions between linear-dendritic copolymers and carbon nanotubes lead to liposome-like nanocapsules. J Mater Chem. 2012;22(14):6947-6952.

72. Ji Z, Lin G, Lu Q, et al. Targeted therapy of SMMC-7721 liver cancer in vitro and in vivo with carbon nanotubes based drug delivery system. J Colloid Interface Sci. 2012;365(1):143-149.

73. Adeli M, Hakimpoor F, Ashiri M, Kabiri R, Bavadi M. Anticancer drug delivery systems based on noncovalent interactions between carbon nanotubes and linear-dendritic copolymers. Soft Matter. 2011;7(8): 4062-4070.

74. Bhirde AA, Patel V, Gavard J, et al. Targeted killing of cancer cells in vivo and in vitro with EGF-directed carbon nanotube-based drug delivery. ACS Nano. 2009;3(2):307-316.

75. Arlt M, Haase D, Hampel S, et al. Delivery of carboplatin by carbon-based nanocontainers mediates increased cancer cell death Nanotechnology. 2010;21(33):335101.

76. Levi-Polyachenko NH, Merkel EJ, Jones BT, Carroll DL, Stewart JH. Rapid photothermal intracellular drug delivery using multiwalled carbon nanotubes. Mol Pharm. 2009;6(4):1092-1099.

77. Slowing II, Vivero-Escoto JL, Wu C-W, Lin VSY. Mesoporous silica nanoparticles as controlled release drug delivery and gene transfection carriers. Adv Drug Deliv Rev. 2008;60(11):1278-1288.

78. Li Y, Li N, Pan W, Yu Z, Yang L, Tang B. Hollow mesoporous silica nanoparticles with tunable structures for controlled drug delivery. ACS Appl Mater Interfaces. 2017;9(3):2123-2129.
79. Wang Y, Zhao Q, Han N, et al. Mesoporous silica nanoparticles in drug delivery and biomedical applications. Nanomedicine. 2015; 11(2):313-327.

80. Lu J, Liong M, Sherman S, et al. Mesoporous silica nanoparticles for cancer therapy: energy-dependent cellular uptake and delivery of paclitaxel to cancer cells. Nanobiotechnology. 2007;3(2):89-95.

81. Lu J, Liong M, Zink JI, Tamanoi F. Mesoporous silica nanoparticles as a delivery system for hydrophobic anticancer drugs. Small. 2007; 3(8):1341-1346.

82. Lebold T, Jung C, Michaelis J, Brauchle C. Nanostructured silica materials as drug-delivery systems for Doxorubicin: single molecule and cellular studies. Nano Lett. 2009;9(8):2877-2883.

83. Rosenholm JM, Peuhu E, Bate-Eya LT, Eriksson JE, Sahlgren C, Linden M Cancer-cell-specific induction of apoptosis using mesoporous silica nanoparticles as drug-delivery vectors. Small. 2010;6(11):1234-1241.

84. Xia T, Kovochich M, Liong M, et al. Polyethyleneimine coating enhances the cellular uptake of mesoporous silica nanoparticles and allows safe delivery of siRNA and DNA constructs. ACS Nano. 2009; 3(10):3273-3286.

85. Prabhakar N, Zhang J, Desai D, et al. Stimuli-responsive hybrid nanocarriers developed by controllable integration of hyperbranched PEI with mesoporous silica nanoparticles for sustained intracellular siRNA delivery. Int J Nanomedicine. 2016;11:6591-6608.

86. Desai D, Zhang J, Sandholm J, et al. Lipid bilayer-gated mesoporous silica nanocarriers for tumor-targeted delivery of zoledronic acid in vivo. Mol Pharm. 2017;14(9):3218-3227.

87. Han N, Zhao Q, Wan L, et al. Hybrid lipid-capped mesoporous silica for stimuli-responsive drug release and overcoming multidrug resistance. ACS Appl Mater Interfaces. 2015;7(5):3342-3351.

88. Haley B, Frenkel E. Nanoparticles for drug delivery in cancer treatment. Urol Oncol Semin Orig Investig. 2008;26(1):57-64.

89. Torchilin V. Tumor delivery of macromolecular drugs based on the EPR effect. Adv Drug Deliv Rev. 2011;63(3):131-135.

90. Torchilin VP. Targeted pharmaceutical nanocarriers for cancer therapy and imaging. AAPS J. 2007;9(2):E128-E147.

91. Pelicano H, Martin D, Xu R, Huang P. Glycolysis inhibition for anticancer treatment. Oncogene. 2006;25(34):4633-4646.

92. Deryugina EI, Quigley JP. Matrix metalloproteinases and tumor metastasis. Cancer Metastasis Rev. 2006;25(1):9-34.

93. Jain RK, Stylianopoulos T. Delivering nanomedicine to solid tumors Nat Rev Clin Oncol. 2010;7(11):653-664.

94. Bae YH, Park K. Targeted drug delivery to tumors: myths, reality and possibility. J Control Release. 2011;153(3):198-205.

95. Danhier F, Feron O, Préat V. To exploit the tumor microenvironment: passive and active tumor targeting of nanocarriers for anti-cancer drug delivery. J Control Release. 2010;148(2):135-146.

96. Patil Y, Sadhukha T, Ma L, Panyam J. Nanoparticle-mediated simultaneous and targeted delivery of paclitaxel and tariquidar overcomes tumor drug resistance. J Control Release. 2009;136(1):21-29.

97. Allen TM. Ligand-targeted therapeutics in anticancer therapy. Nat Rev Cancer. 2002;2(10):750-763.

98. Pirollo KF, Chang EH. Does a targeting ligand influence nanoparticle tumor localization or uptake? Trends Biotechnol. 2008;26(10): 552-558.

99. Sudimack J, Lee RJ. Targeted drug delivery via the folate receptor. Adv Drug Deliv Rev. 2000;41(2):147-162.

100. Gan CW, Feng S-S. Transferrin-conjugated nanoparticles of Poly(lactide)-d- $\alpha$-Tocopheryl polyethylene glycol succinate diblock copolymer for targeted drug delivery across the blood-brain barrier. Biomaterials. 2010;31(30):7748-7757.

101. Neri D, Bicknell R. Tumour vascular targeting. Nat Rev Cancer. 2005; 5(6):436-446.

102. Martinez-Carmona M, Colilla M, Vallet-Regi M. Smart mesoporous nanomaterials for antitumor therapy. Nanomaterials. 2015;5(4): 1906-1937.

103. Choi J-S, Park J-S. Development of docetaxel nanocrystals surface modified with transferrin for tumor targeting. Drug Des Devel Ther. 2017;11:17-26. 
104. Song Z, Lin Y, Zhang X, et al. Cyclic RGD peptide-modified liposomal drug delivery system for targeted oral apatinib administration: enhanced cellular uptake and improved therapeutic effects. Int $J$ Nanomedicine. 2017;12:1941-1958.

105. Wu W, Zheng Y, Wang R, et al. Antitumor activity of folate-targeted, paclitaxel-loaded polymeric micelles on a human esophageal EC9706 cancer cell line. Int J Nanomedicine. 2012;7:3487-3502.

106. Hanahan D, Weinberg RA. Hallmarks of cancer: the next generation. Cell. 2011;144(5):646-674.

107. Hall JE. Guyton and Hall Textbook of Medical Physiology. Philadelphia, PA: Elsevier Health Sciences; 2015.

108. Torre LA, Bray F, Siegel RL, Ferlay J, Lortet-Tieulent J, Jemal A. Global cancer statistics, 2012. CA Cancer J Clin. 2015;65(2):87-108.

109. Anderson D, Najafzadeh M, Gopalan R, et al. Sensitivity and specificity of the empirical lymphocyte genome sensitivity (LGS) assay: implications for improving cancer diagnostics. FASEB J. 2014;28(10): 4563-4570.

110. Meyerson M, Gabriel S, Getz G. Advances in understanding cancer genomes through second-generation sequencing. Nat Rev Genet. 2010; 11(10):685-696.

111. Singh $P$, Singh A. Ocular adverse effects of anti-cancer chemotherapy. $J$ Cancer Ther Res. 2012;1(1):5.

112. Aravind A, Varghese SH, Veeranarayanan S, et al. Aptamer-labeled PLGA nanoparticles for targeting cancer cells. Cancer Nanotechnol. 2012;3(1-6):1-12.

113. Abner AL, Recht A, Eberlein T, et al. Prognosis following salvage mastectomy for recurrence in the breast after conservative surgery and radiation therapy for early-stage breast cancer. J Clin Oncol. 1993; 11(1):44-48

114. Lichter AS, Lippman ME, Danforth D, et al. Mastectomy versus breast-conserving therapy in the treatment of stage I and II carcinoma of the breast: a randomized trial at the National Cancer Institute. J Clin Oncol. 1992;10(6):976-983.

115. Bonadonna G, Valagussa P, Moliterni A, Zambetti M, Brambilla C. Adjuvant cyclophosphamide, methotrexate, and fluorouracil in nodepositive breast cancer - the results of 20 years of follow-up. $N$ Engl J Med. 1995;332(14):901-906

116. Zuur CL, Simis YJ, Verkaik RS, et al. Hearing loss due to concurrent daily low-dose cisplatin chemoradiation for locally advanced head and neck cancer. Radiother Oncol. 2008;89(1):38-43.

117. Adair JH, Parette MP, Altınoglu EI, Kester M. Nanoparticulate alternatives for drug delivery. ACS Nano. 2010;4(9):4967-4970.

118. Chawla JS, Amiji MM. Biodegradable poly ( $\varepsilon$-caprolactone) nanoparticles for tumor-targeted delivery of tamoxifen. Int J Pharm. 2002; 249(1):127-138.

119. Potineni A, Lynn DM, Langer R, Amiji MM. Poly (ethylene oxide)modified poly ( $\beta$-amino ester) nanoparticles as a $\mathrm{pH}$-sensitive biodegradable system for paclitaxel delivery. J Control Release. 2003;86(2): 223-234.

120. Shenoy DB, Amiji MM. Poly (ethylene oxide)-modified poly ( $\varepsilon$-caprolactone) nanoparticles for targeted delivery of tamoxifen in breast cancer. Int J Pharm. 2005;293(1):261-270.

121. Milane L, Duan Z, Amiji M. Development of EGFR-targeted polymer blend nanocarriers for combination paclitaxel/lonidamine delivery to treat multi-drug resistance in human breast and ovarian tumor cells. Mol Pharm. 2010;8(1):185-203.

122. Cohen SM, Mukerji R, Cai S, Damjanov I, Forrest ML, Cohen MS. Subcutaneous delivery of nanoconjugated doxorubicin and cisplatin for locally advanced breast cancer demonstrates improved efficacy and decreased toxicity at lower doses than standard systemic combination therapy in vivo. Am J Surg. 2011;202(6):646-653.

123. Siegel RL, Miller KD, Jemal A. Cancer statistics, 2017. CA Cancer J Clin. 2017;67(1):7-30.

124. Abou-Alfa GK, Letourneau R, Harker G, et al. Randomized phase III study of exatecan and gemcitabine compared with gemcitabine alone in untreated advanced pancreatic cancer. J Clin Oncol. 2006;24(27): $4441-4447$.
125. Barenholz YC. Doxil ${ }^{\circledR}$ - the first FDA-approved nano-drug: lessons learned. J Control Release. 2012;160(2):117-134.

126. Cabral H, Murakami M, Hojo H, et al. Targeted therapy of spontaneous murine pancreatic tumors by polymeric micelles prolongs survival and prevents peritoneal metastasis. Proc Natl Acad Sci USA. 2013;110(28):11397-11402.

127. Conroy T, Desseigne F, Ychou M, et al; Groupe Tumeurs Digestives of Unicancer; PRODIGE Intergroup. FOLFIRINOX versus gemcitabine for metastatic pancreatic cancer. N Engl J Med. 2011;364(19): 1817-1825.

128. Leamon CP, Lovejoy CD, Nguyen B. Patient selection and targeted treatment in the management of platinum-resistant ovarian cancer. Pharmgenomics Pers Med. 2013;6(1):113-125.

129. Lee GY, Qian WP, Wang L, et al. Theranostic nanoparticles with controlled release of gemcitabine for targeted therapy and MRI of pancreatic cancer. ACS Nano. 2013;7(3):2078-2089.

130. Mir M, Ishtiaq S, Rabia S, et al. Nanotechnology: from in vivo imaging system to controlled drug delivery. Nanoscale Res Lett. 2017; 12(1):500.

131. Ferrari M. Cancer nanotechnology: opportunities and challenges. Nat Rev Cancer. 2005;5(3):161-171.

132. Vincent A, Herman J, Schulick R, Hruban RH, Goggins M. Pancreatic cancer. Lancet. 2011;378(9791):607-620.

133. Von Hoff DD, Ramanathan RK, Borad MJ, et al. Gemcitabine plus nab-paclitaxel is an active regimen in patients with advanced pancreatic cancer: a phase I/II trial. J Clin Oncol. 2011;29(34): 4548-4554.

134. Davis ME, Zuckerman JE, Choi CHJ, et al. Evidence of RNAi in humans from systemically administered siRNA via targeted nanoparticles. Nature. 2010;464(7291):1067-1070.

135. Su J, Baigude H, McCarroll J, Rana TM. Silencing microRNA by interfering nanoparticles in mice. Nucleic Acids Res. 2011;39(6):e38.

136. Schroeder A, Levins CG, Cortez C, Langer R, Anderson DG. Lipidbased nanotherapeutics for siRNA delivery. J Intern Med. 2010;267(1): 9-21.

137. Pirollo KF, Rait A, Zhou Q, et al. Materializing the potential of small interfering RNA via a tumor-targeting nanodelivery system. Cancer Res. 2007;67(7):2938-2943.

138. Tsai CJ, Lu DK. Small colorectal polyps: histopathology and clinical significance. Am J Gastroenterol. 1995;90(6):988-994.

139. Chuah LH, Billa N, Roberts CJ, Burley JC, Manickam S. Curcumincontaining chitosan nanoparticles as a potential mucoadhesive delivery system to the colon. Pharm Dev Technol. 2013;18(3):591-599.

140. Cohen MH, Gootenberg J, Keegan P, Pazdur R. FDA drug approval summary: bevacizumab plus FOLFOX4 as second-line treatment of colorectal cancer. Oncologist. 2007;12(3):356-361.

141. Lagarce F, Cruaud O, Deuschel C, Bayssas M, Griffon-Etienne G, Benoit J. Oxaliplatin loaded PLAGA microspheres: design of specific release profiles. Int J Pharm. 2002;242(1):243-246.

142. Liu K, Wang ZQ, Wang SJ, et al. Hyaluronic acid-tagged silica nanoparticles in colon cancer therapy: therapeutic efficacy evaluation. Int J Nanomedicine. 2015;10:6445-6454.

143. Muthoosamy K, Abubakar IB, Bai RG, Loh HS, Manickam S. Exceedingly higher co-loading of curcumin and paclitaxel onto polymerfunctionalized reduced graphene oxide for highly potent synergistic anticancer treatment. Sci Rep. 2016;6:32808.

144. Cutler DM. Are we finally winning the war on cancer? JEcon Perspect. 2008;22(4):3-26.

145. Peng G, Tisch U, Adams O, et al. Diagnosing lung cancer in exhaled breath using gold nanoparticles. Nat Nanotechnol. 2009;4(10): 669-673.

146. Tomoda K, Ohkoshi T, Hirota K, et al. Preparation and properties of inhalable nanocomposite particles for treatment of lung cancer. Colloids Surf B Biointerfaces. 2009;71(2):177-182.

147. Azarmi S, Tao X, Chen H, et al. Formulation and cytotoxicity of doxorubicin nanoparticles carried by dry powder aerosol particles. Int J Pharm. 2006;319(1-2):155-161. 
148. Jinturkar KA, Anish C, Kumar MK, Bagchi T, Panda AK, Misra AR. Liposomal formulations of Etoposide and Docetaxel for p53 mediated enhanced cytotoxicity in lung cancer cell lines. Biomaterials. 2012;33(8):2492-2507.

149. Koshkina NV, Kleinerman ES, Waldrep C, et al. 9-Nitrocamptothecin liposome aerosol treatment of melanoma and osteosarcoma lung metastases in mice. Clin Cancer Res. 2000;6(7):2876-2880.

150. Lu Z, Yeh T-K, Wang J, et al. Paclitaxel gelatin nanoparticles for intravesical bladder cancer therapy. J Urol. 2011;185(4):1478-1483.

151. Tseng C-L, Su W-Y, Yen K-C, Yang K-C, Lin F-H. The use of biotinylated-EGF-modified gelatin nanoparticle carrier to enhance cisplatin accumulation in cancerous lungs via inhalation. Biomaterials. 2009;30(20):3476-3485.

152. Cao F, Ding B, Sun M, Guo C, Zhang L, Zhai G. Lung-targeted delivery system of curcumin loaded gelatin microspheres. Drug Deliv. 2011;18(8):545-554.

153. Karthikeyan S, Prasad NR, Ganamani A, Balamurugan E. Anticancer activity of resveratrol-loaded gelatin nanoparticles on NCI-H460 nonsmall cell lung cancer cells. Biomed Prev Nutr. 2013;3(1):64-73.

154. Cascone MG, Lazzeri L, Carmignani C, Zhu Z. Gelatin nanoparticles produced by a simple $\mathrm{W} / \mathrm{O}$ emulsion as delivery system for methotrexate. J Mater Sci Mater Med. 2002;13(5):523-526.

155. Taratula $\mathrm{O}$, Garbuzenko OB, Chen AM, Minko T. Innovative strategy for treatment of lung cancer: targeted nanotechnology-based inhalation co-delivery of anticancer drugs and siRNA. J Drug Target. 2011; 19(10):900-914.

156. Taratula O, Kuzmov A, Shah M, Garbuzenko OB, Minko T. Nanostructured lipid carriers as multifunctional nanomedicine platform for pulmonary co-delivery of anticancer drugs and siRNA. J Control Release. 2013;171(3):349-357.

157. Patlolla RR, Chougule M, Patel AR, Jackson T, Tata PN, Singh M. Formulation, characterization and pulmonary deposition of nebulized celecoxib encapsulated nanostructured lipid carriers. J Control Release. 2010;144(2):233-241.

158. Hitzman CJ, Wattenberg LW, Wiedmann TS. Pharmacokinetics of 5-fluorouracil in the hamster following inhalation delivery of lipidcoated nanoparticles. J Pharm Sci. 2006;95(6):1196-1211.

159. Kim JH, Kim Y, Bae KH, Park TG, Lee JH, Park K. Tumor-targeted delivery of paclitaxel using low density lipoprotein-mimetic solid lipid nanoparticles. Mol Pharm. 2015;12(4):1230-1241.

160. Patro NM, Devi K, Pai RS, Suresh S. Evaluation of bioavailability, efficacy, and safety profile of doxorubicin-loaded solid lipid nanoparticles. J Nanopart Res. 2013;15(12):1-18.

161. Yassin AE, Anwer MK, Mowafy HA, El-Bagory IM, Bayomi MA, Alsarra IA. Optimization of 5-fluorouracil solid-lipid nanoparticles: a preliminary study to treat colon cancer. Int J Med Sci. 2010;7(6): 398-408.
162. Khan DR, Webb MN, Cadotte TH, Gavette MN. Use of targeted liposome-based chemotherapeutics to treat breast cancer. Breast Cancer. 2015;9(suppl 2):1.

163. Park JW. Liposome-based drug delivery in breast cancer treatment. Breast Cancer Res. 2002;4(3):1.

164. Abu-Rmaileh R, Attwood D, D'Emanuele A. Dendrimers in cancer therapy. Drug Deliv Syst Sci. 2003;3(3):65-70.

165. Sampathkumar S-G, Yarema KJ. Targeting cancer cells with dendrimers. Chem Biol. 2005;12(1):5-6.

166. Ganta S, Devalapally H, Shahiwala A, Amiji M. A review of stimuliresponsive nanocarriers for drug and gene delivery. J Control Release. 2008;126(3):187-204.

167. Guo J, Gao X, Su L, et al. Aptamer-functionalized PEG-PLGA nanoparticles for enhanced anti-glioma drug delivery. Biomaterials. 2011; 32(31):8010-8020.

168. Al Faraj A, Shaik AS, Ratemi E, Halwani R. Combination of drugconjugated SWCNT nanocarriers for efficient therapy of cancer stem cells in a breast cancer animal model. J Control Release. 2016;225: 240-251

169. Dhar S, Liu Z, Thomale J, Dai H, Lippard SJ. Targeted single-wall carbon nanotube-mediated $\mathrm{Pt}(\mathrm{IV})$ prodrug delivery using folate as a homing device. J Am Chem Soc. 2008;130(34):11467-11476.

170. Heister E, Neves V, Tîlmaciu C, et al. Triple functionalisation of singlewalled carbon nanotubes with doxorubicin, a monoclonal antibody, and a fluorescent marker for targeted cancer therapy. Carbon $N Y$. 2009;47(9):2152-2160.

171. Liu Z, Chen K, Davis C, et al. Drug delivery with carbon nanotubes for in vivo cancer treatment. Cancer Res. 2008;68(16):6652-6660.

172. Lu Y-J, Wei K-C, Ma C-CM, Yang S-Y, Chen J-P. Dual targeted delivery of doxorubicin to cancer cells using folate-conjugated magnetic multi-walled carbon nanotubes. Colloids Surf B Biointerfaces. 2012;89:1-9.

173. Samori C, Ali-Boucetta H, Sainz R, et al. Enhanced anticancer activity of multi-walled carbon nanotube-methotrexate conjugates using cleavable linkers. Chem Commun. 2010;46(9):1494-1496.

174. Zhang X, Meng L, Lu Q, Fei Z, Dyson PJ. Targeted delivery and controlled release of doxorubicin to cancer cells using modified single wall carbon nanotubes. Biomaterials. 2009;30(30):6041-6047.

175. Singh P, Destito G, Schneemann A, Manchester M. Canine parvovirus-like particles, a novel nanomaterial for tumor targeting. J Nanobiotechnology. 2006;4:2-12.
International Journal of Nanomedicine

\section{Publish your work in this journal}

The International Journal of Nanomedicine is an international, peerreviewed journal focusing on the application of nanotechnology in diagnostics, therapeutics, and drug delivery systems throughout the biomedical field. This journal is indexed on PubMed Central, MedLine, CAS, SciSearch $®$, Current Contents $\AA /$ Clinical Medicine,

\section{Dovepress}

Journal Citation Reports/Science Edition, EMBase, Scopus and the Elsevier Bibliographic databases. The manuscript management system is completely online and includes a very quick and fair peer-review system, which is all easy to use. Visit http://www.dovepress.com/ testimonials.php to read real quotes from published authors. 